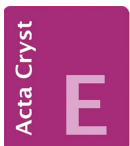

CRYSTALLOGRAPHIC COMMUNICATIONS

ISSN 2056-9890

Received 9 January 2022

Accepted 19 January 2022

‡ Deceased

Keywords: crystal structure; lanthanide complexes; luminescence; antenna ligands.

CCDC reference: 2143190

Supporting information: this article has supporting information at journals.iucr.org/e

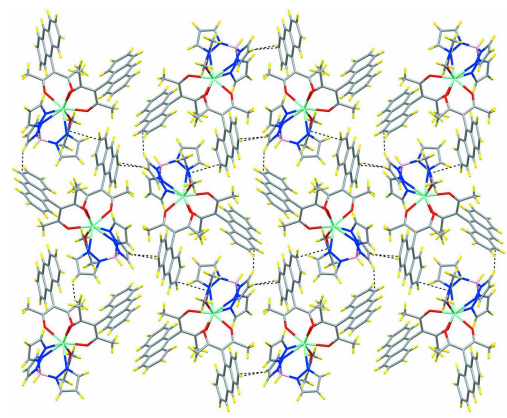

\section{Bis[3-(anthracen-9-yl)pentane-2,4-dionato- $\left.\kappa^{2} O, O^{\prime}\right]$ - $(\mathrm{N}, \mathrm{N}$-dimethylformamide- $\kappa \mathrm{O})$ [tris(pyrazol-1-yl- $\left.\kappa N^{2}\right)$ hydroborato]europium(III)}

\author{
Elena A. Mikhalyova, ${ }^{a *}$ Matthias Zeller, ${ }^{\text {b Jerry } P .}$ Jasinski, ${ }^{c} \neq$ Manpreet Kaur $^{\mathrm{c}}$ and \\ Anthony W. Addison ${ }^{\mathrm{d}}$
}

aL. V. Pisarzhevskii Institute of Physical Chemistry of the National Academy of Sciences of Ukraine, Prospekt Nauki 31, Kyiv, 03028, Ukraine, 'bepartment of Chemistry, Purdue University, 560 Oval Drive, West Lafayette, IN, 47907, USA, ${ }^{\mathbf{c}}$ Department of Chemistry, Keene State College, Keene, NH 03435, USA, and ${ }^{\mathbf{d}}$ Department of Chemistry, Drexel University, Philadelphia, PA 19104-2816, USA. *Correspondence e-mail: elenaamikhalyova@gmail.com

The title compound, $\left[\mathrm{Eu}\left(\mathrm{C}_{9} \mathrm{H}_{10} \mathrm{BN}_{6}\right)\left(\mathrm{C}_{19} \mathrm{H}_{15} \mathrm{O}_{2}\right)_{2}\left(\mathrm{C}_{3} \mathrm{H}_{7} \mathrm{NO}\right)\right]$ or [ $\mathrm{TpEu}($ Anthracac $\left.)_{2}(\mathrm{DMF})\right]$, was synthesized by reaction of a tris(pyrazolyl)borate $\left(\mathrm{Tp}^{-}\right) \mathrm{Eu}^{3+}$ complex with 3-(anthracen-9-yl)pentane-2,4-dione (HAnthracac) in the presence of triethylamine. In the title compound, $\mathrm{Eu}^{3+}$ is located in an octavertex square-pyramidal coordination environment. In the two Anthracac ${ }^{-}$ ligands, the anthracene and nearly planar acetylacetonate fragments are almost orthogonal. Neighboring molecules of $\mathrm{TpEu}(\text { Anthracac })_{2}(\mathrm{DMF})$ are connected in the crystal by intermolecular van der Waals interactions, while $\pi$-stacking interactions are limited to the edges of two anthracene rings.

\section{Chemical context}

Lanthanide complexes find numerous applications as, for example, luminescent materials, markers, security inks, components of lasers, light-emitting diodes, and many others (Bünzli, 2017; Venturini Filho et al., 2018; Khullar et al., 2019; Bünzli, 2019). This variety of uses relies in large parts on the electronic structure of the $\mathrm{Ln}^{3+}$ ions, which leads to electronic transitions occurring between $f$-orbitals, providing them with unique luminescence characteristics, including high color purity and exact reproducibility of the emitted light color (Sarkar et al., 2019; Wang, Pu et al., 2019; Wang, Zhao et al., 2019). In spite of these advantages, the electronic structure of $\mathrm{Ln}^{3+}$ ions causes the luminescence to be of low intensity due to the forbidden nature of $f-f$ electronic transitions (Bünzli, 2017; Zhang et al., 2020; Wang, Zhao et al., 2019), hence the weak absorbance of the exciting radiation. This feature is usually evaded by using organic 'antenna' ligands, which are capable of absorbing exciting radiation and transferring the gained energy to the $\mathrm{Ln}^{3+}$ ions (Bünzli, 2017; Carneiro Neto et al., 2019; Aulsebrook et al., 2018). Recently, it was shown (Mikhalyova et al., 2017; Gheno et al., 2014; Mikhalyova et al., 2020; Bortoluzzi et al., 2012) that tris(pyrazolyl)borate anions are efficient antenna ligands for $\mathrm{Tb}^{3+}$ and $\mathrm{Eu}^{3+}$, both exhibiting emission in the visible range. Anions of $\beta$-diketones with different substituents are also well-known antenna ligands (Wang, Zhao et al., 2019; Nehra et al., 2022). To increase the extinction coefficients of the ligands, it can be of advantage to add a large conjugated moiety to their structure. Recently it was found by us (Kandel et al., 2017; Mikhalyova et 
al., 2017), that the combination of several antenna ligands in one compound can have complex and unpredictable effects on its luminescence characteristics, which also depend on the molecular and crystal structure details of the complex. Thus, for this work, an $\mathrm{Eu}^{3+}$ complex with two types of antenna ligands, i.e. tris(pyrazolyl)borate $\left(\mathrm{Tp}^{-}\right)$and 3-(anthracen-9yl)pentane-2,4-dionate (Anthracac ${ }^{-}$), of the composition $\mathrm{TpEu}(\text { Anthracac })_{2}(\mathrm{DMF})$ was obtained and its molecular and crystal structures were studied.

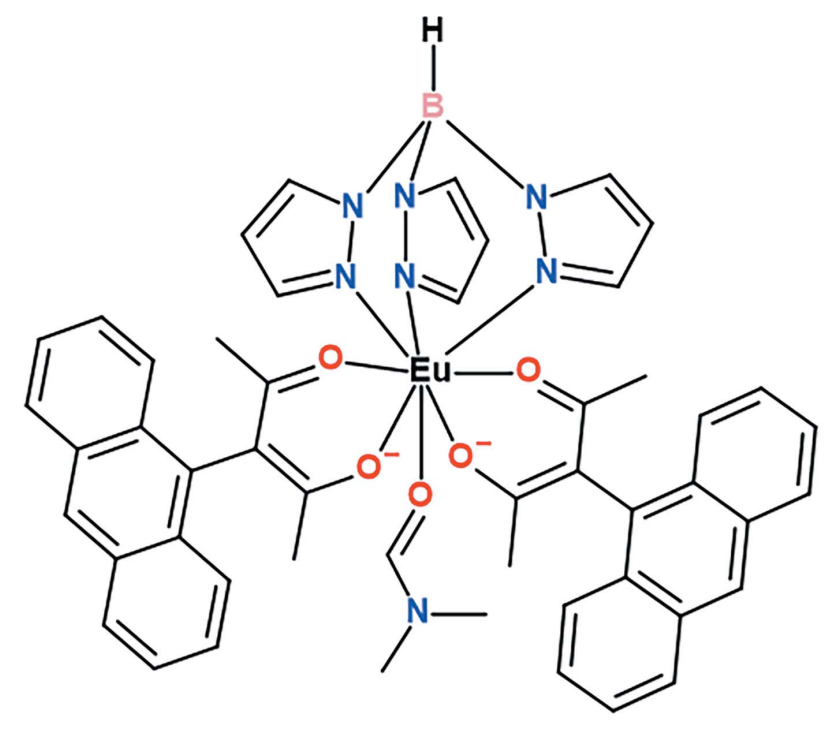

\section{Structural commentary}

The title compound is a neutral metal-containing complex and crystallizes in the monoclinic $P 2_{1} / n$ space group with only one molecule in the asymmetric unit (Fig. 1). The unit cell contains two molecules of each enantiomer, whose crystallographic positions are related by the inversion centers, glide planes and screw axes (Fig. 2). The asymmetric unit consists of the $\mathrm{Eu}^{3+}$

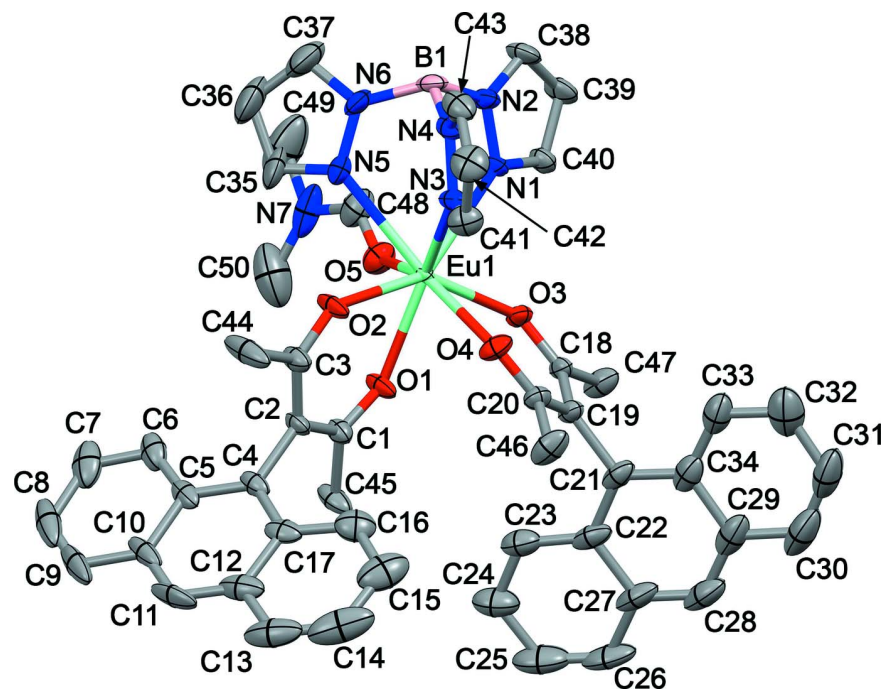

Figure 1

The molecular structure of the title compound. Atomic displacement ellipsoids are drawn at the $50 \%$ probability level. $\mathrm{H}$ atoms are omitted for clarity of presentation.

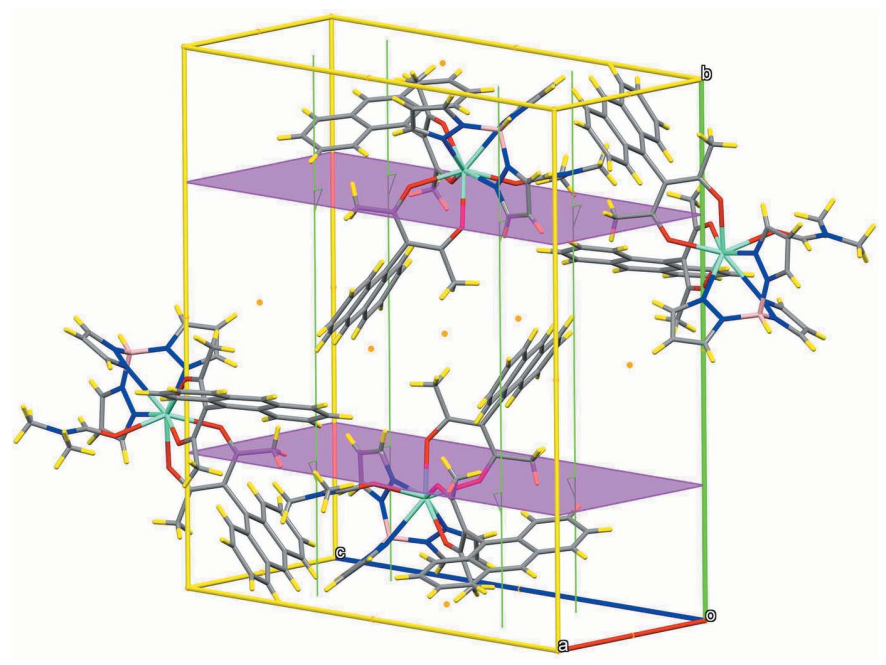

Figure 2

Stick diagram of a unit-cell view with symmetry elements: inversion centers (orange), glide planes (violet) and screw axes (green).

ion surrounded by one $\mathrm{Tp}^{-}$and two Anthracac ${ }^{-}$ligands and one dimethylformamide molecule. Of these ligands, the $\mathrm{Tp}^{-}$is coordinated tridentately, donating three $\mathrm{N}$ atoms to the coordination polyhedron, while each Anthracac ${ }^{-}$acts as a bidentate $\mathrm{O}$ ligand, donating a combined four $\mathrm{O}$ atoms. The DMF molecule acts as a unidentate $\mathrm{O}$ donor. As is typical for lanthanide ions with seven, eight or nine coordinating atoms, the assignment of the coordination geometry carries some ambiguity. Several different criteria have been proposed to define the shape of such a coordination polyhedron. Use of the Shape 2.1 software (Casanova et al., 2005; Alvarez et al., 2005), indicates that the $\mathrm{Eu}^{3+}$ ion in the title compound is an octavertex with a slightly distorted square-antiprismatic geometry (Fig. 3), with a mean angle between the capping and basal square planes of the coordination polyhedron of $0.75(8)^{\circ}$. According to the Lippard \& Russ (1968) criterion, the angle between the body-diagonal trapezoids for the title compound, $\omega$, is $88.24(7)^{\circ}$, which is closer to the angle for a dodecahedron $\left(90.0^{\circ}\right)$ than a square antiprism $\left(79.3^{\circ}\right)$. A more accurate criterion is the one proposed by Porai-Koshits and Aslanov

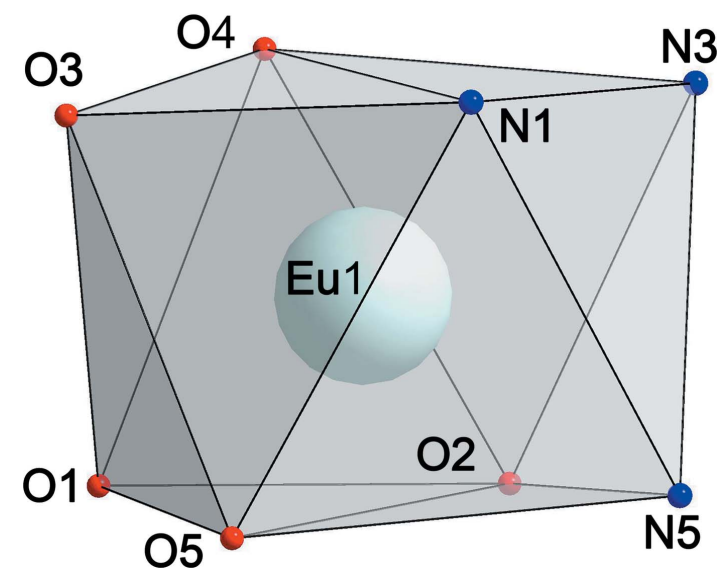

Figure 3

The geometry of the $\mathrm{Eu}^{3+}$ coordination polyhedron. 


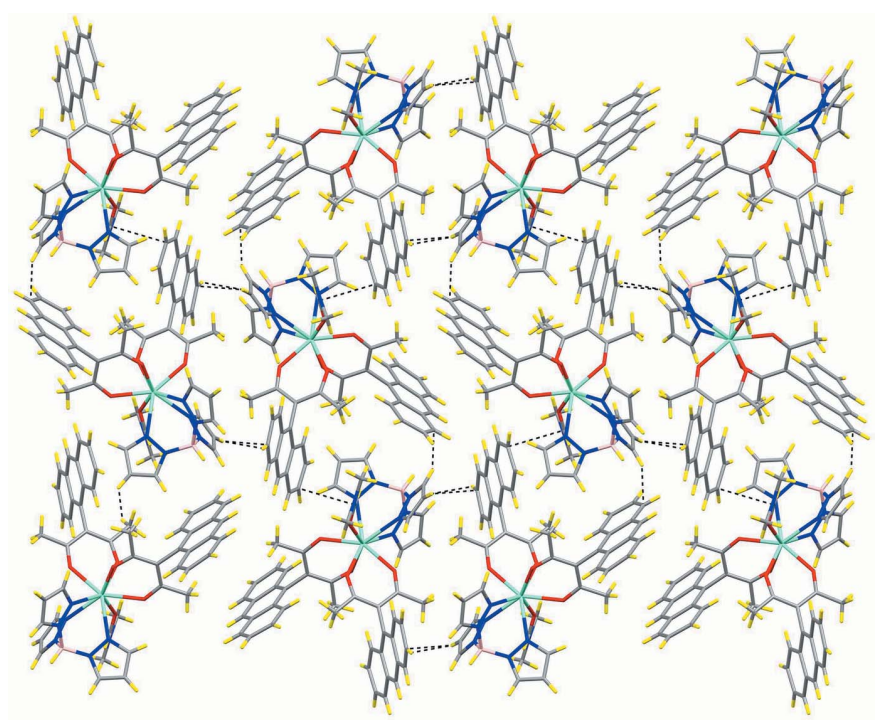

Figure 4

Packing view along the $a$-axis (see also Fig. S3).

(1972) based on the angles, $\delta$, between pairs of faces intersecting along the edges connecting the vertices where the five edges intersect. The respective angles for the complex here are $6.6(1), 8.9(1), 43.3(1)$, and $49.7(1)^{\circ}$ and the degrees of nonplanarity of the diagonal trapezoids, $\varphi$, are 18.81 (9) and $19.74(1)^{\circ}$. From these criteria, the $\delta$ angles are closer to those of an idealized square antiprism, yet the $\varphi$ angles correspond to those of a bicapped trigonal prism. Thus, three different criteria define three different polyhedra and among these criteria, only the $\delta$-based one agrees with the assignment using the Shape 2.1 software.

The lengths for Eu-donor atom bonds are listed in Table 1 and these are in the usual range for compounds with similar ligands (Mikhalyova et al., 2020; Lawrence et al., 2001; Dei et al., 2000).

Regarding the geometrical features of the ligands, it should be noted that the planar anthracene moiety and the nearly planar acetylacetonate fragment are almost orthogonal to
Table 1

Selected bond lengths $(\AA)$.

\begin{tabular}{llll}
\hline Eu1-O1 & $2.351(3)$ & Eu1-O5 & $2.417(3)$ \\
Eu1-O2 & $2.313(3)$ & Eu1-N1 & $2.582(3)$ \\
Eu1-O3 & $2.344(3)$ & Eu1-N3 & $2.555(3)$ \\
Eu1-O4 & $2.340(3)$ & Eu1-N5 & $2.573(3)$ \\
\hline
\end{tabular}

Table 2

Selected intermolecular interatomic distances $(\AA)$.

\begin{tabular}{llll}
\hline $\mathrm{C} 8 \cdots \mathrm{C} 15^{\mathrm{i}}$ & $3.258(8)$ & $\mathrm{C} 8 \cdots \mathrm{H} 36^{\mathrm{ii}}$ & 2.698 \\
\hline $\mathrm{H} 8 \cdots \mathrm{C} 15^{\mathrm{i}}$ & 2.817 & $\mathrm{H} 37 \cdots \mathrm{C} 26^{\mathrm{iii}}$ & 2.830 \\
$\mathrm{H} 50 C \cdots \mathrm{N} 3^{\mathrm{i}}$ & 2.680 & $\mathrm{C} 48 \cdots \mathrm{C} 14^{\mathrm{iii}}$ & $3.159(8)$ \\
$\mathrm{H} 50 \mathrm{C} \cdots \mathrm{C} 41^{\mathrm{i}}$ & 2.718 & & \\
\hline
\end{tabular}

Symmetry codes: (i) $-1+x, y, z$; (ii) $x, 2-y, 1-z$; (iii) $-\frac{1}{2}+x, \frac{3}{2}-y,-\frac{1}{2}+z$.

each other in each Anthracac ${ }^{-}$ligand, subtending dihedral angles of 87.84 (7) and $79.98(7)^{\circ}$. This is due to the presence of the $\mathrm{CH}_{3}$ groups, which prevent rotation of the anthracenyl fragments along the $\mathrm{C} 2-\mathrm{C} 4$ and $\mathrm{C} 19-\mathrm{C} 21$ bonds.

\section{Supramolecular features}

The crystal packing of the title compound consists of separate neutral molecules. Several short contacts are observed (Table 2), but none of these exhibit the typical characteristics of directional attractive interactions, i.e. they are not hydrogen bonds or $\mathrm{C}-\mathrm{H} \cdots \pi$ interactions. It thus can be said that these molecules are organized in the lattice predominantly by intermolecular van der Waals or dispersion interactions (Fig. 4, Table 2).

$\pi$-Stacking interactions play no dominant role in this structure. For one of the anthracene fragments (C4-C16) no $\pi-\pi$ stacking interactions are observed at all. For the other anthracenyl group (C21-C34) one $\pi$-interaction is present, but it is limited to part of one of the outer phenylene groups, C29$\mathrm{C} 34$, which is $\pi$-stacked with its inversion-related counterpart [symmetry code: (i) $2-x, 1-y, 1-z$ ], with a centroid-tocentroid distance of 3.958 (8) $\AA$ (Fig. 5). The remainder of the (a)

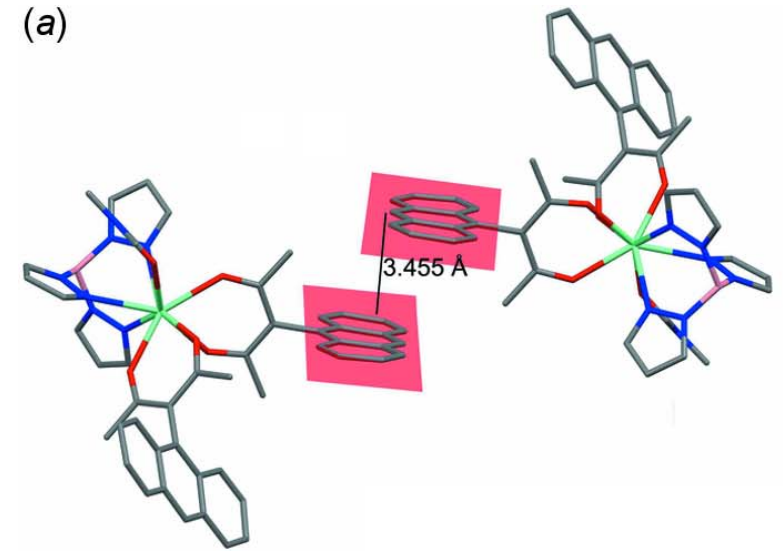

(b)

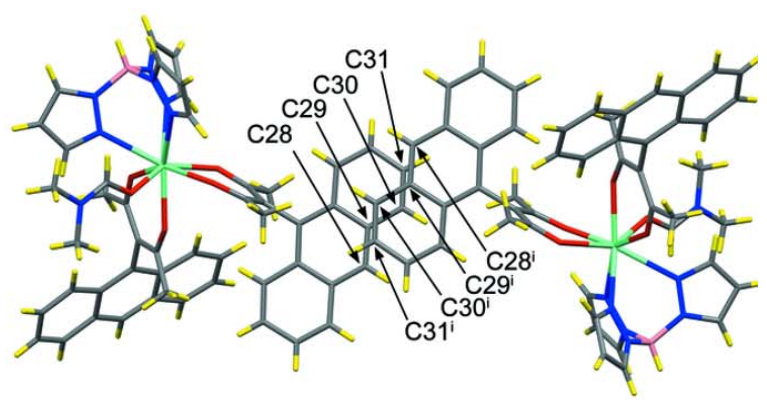

Figure 5

(a) View of the $\pi-\pi$ stacking interaction observed for one of the phenylene groups of the anthracene fragments and $(b)$ a view of the same, perpendicular to the planes of the anthracenyl $(\mathrm{C} 21-\mathrm{C} 34)$ fragments [symmetry code: (i) $2-x, 1-y, 1-z$ ]. Other occurrences of parallel (but not stacked) anthracenyl units are shown in Figs. S1, S2 and S4. 
Table 3

Experimental details.

Crystal data

Chemical formula

$M_{\mathrm{r}}$

Crystal system, space group

Temperature (K)

$a, b, c(\AA)$

$\beta\left({ }^{\circ}\right)$

$V\left(\AA^{3}\right)$

$Z$

Radiation type

$\mu\left(\mathrm{mm}^{-1}\right)$

Crystal size (mm)

Data collection

Diffractometer

Absorption correction

$T_{\min }, T_{\max }$

No. of measured, independent and

observed $[I>2 \sigma(I)]$ reflections

$R_{\text {int }}$

$(\sin \theta / \lambda)_{\max }\left(\AA^{-1}\right)$

$\left[\mathrm{Eu}\left(\mathrm{C}_{9} \mathrm{H}_{10} \mathrm{BN}_{6}\right)\left(\mathrm{C}_{19} \mathrm{H}_{15} \mathrm{O}_{2}\right)_{2}\left(\mathrm{C}_{3} \mathrm{H}_{7}\right.\right.$ $\mathrm{NO})]$

988.71

Monoclinic, $P 2_{1} / n$

173

9.3728 (3), 22.5555 (7), 22.0840 (6)

$96.314(3)$

4640.4 (2)

4

$\mathrm{Cu} K \alpha$

10.11

$0.48 \times 0.18 \times 0.12$

Rigaku Oxford Diffraction Gemini Eos

Multi-scan (CrysAlis PRO; Rigaku OD, 2015)

$0.163,1.000$

$19671,8850,7261$

0.048

0.615

Refinement

$R\left[F^{2}>2 \sigma\left(F^{2}\right)\right], w R\left(F^{2}\right), S$

No. of reflections

No. of parameters

$\mathrm{H}$-atom treatment

$\Delta \rho_{\max }, \Delta \rho_{\min }\left(\mathrm{e} \AA^{-3}\right)$

$0.042,0.106,1.03$

8850

583

$\mathrm{H}$-atom parameters constrained

$1.09,-1.25$

Computer programs: CrysAlis PRO (Rigaku OD, 2015), SHELXT (Sheldrick, 2015a), SHELXL (Sheldrick, 2015b), OLEX2 (Dolomanov et al., 2009), Mercury (Macrae et al., 2020), and publCIF (Westrip, 2010).

anthracenyl group does not participate in the $\pi-\pi$ stacking interaction; for the entire anthracene moiety $(\mathrm{C} 21-\mathrm{C} 34)$ the distance between the centroids is 6.006 (8) $\AA$. The distance between inversion-related mean planes $\left(\mathrm{C} 21-\mathrm{C} 34\right.$ and $\mathrm{C} 21^{\mathrm{i}}-$ $\left.\mathrm{C} 34^{\mathrm{i}}\right)$ is $3.455 \AA$, indicating a medium strength stacking interaction (Fig. 5).

\section{Database survey}

The Cambridge Structural Database (CSD, version 5.41, updates till Aug 2020; Groom et al., 2016) contains just one crystal structure with an $\mathrm{Ln}^{3+}$ ion surrounded by two $\beta$-diketonate anions and one tris(pyrazolyl)borate ligand, namely, bis(1,3-diphenyl-1,3-propanedionato- $\left.O, O^{\prime}\right)\{$ hydrotris[3-(2pyridyl)-

pyrazol-1-yl]borato\}praseodymium(III) (FOLZUC; Davies et al., 2005). However, in this compound the $\mathrm{Pr}^{3+}$ ion is decacoordinate owing to the presence of 2-pyridyl substituents in the tris(pyrazolyl)borate ligand, so a direct comparison of the coordination geometries of this and the title compound is not possible.

Fragments containing one $L n$ ion surrounded by at least one $\beta$-diketonate anion and one tris(pyrazolyl)borate ligand encompass 34 entities (including FOLZUC). Most of them (28), contain eight-coordinate lanthanide ions with two tris(pyrazolyl)borate ligands and one $\beta$-diketonate anion: DULWEP, DULWIT, DULWOZ, DULWUF, DULXAM,
DULXEQ, DULXIU, DULXOA, DULXUG, DULYAN, DULYER, DULYIV, DULYOB, DULYUH, DULZAO, DULZES, DULZIW, DULZOC, DULZUI and DUMDAT (all Mikhalyova et al., 2020); ESUHOP (Galler et al., 2004); GIFCUT, GIFDAA (Moss et al., 1988); GIFCUT10, GIFDAA10 (Moss et al., 1989); KIFKUI (Guégan et al., 2018); XICHIA (Lawrence et al., 2001). Again, the coordination environment of these compounds and the title one cannot be directly compared. One of the compounds, FOLZUC, is discussed above and another, [tris(3-t-butyl-5-methylpyrazolyl)hydroborato](2,2,6,6-tetramethylheptane-3,5-dionato)ytterbium(II) (ESUJIL; Morissette et al., 2004) is a neutral molecule of $\mathrm{Yb}^{2+}$. Four entities are complexes with salicylaldehyde derivatives [JAJRAO (Onishi et al., 2004), QIDGAL, QIDGAL01 (Onishi et al., 1999), and ZUCCIJ (Lawrence et al., 1996)], which are also $\beta$-diketonate anions, but, again, compounds with these anions contain eightcoordinate $\mathrm{Ln}^{3+}$ ions.

Only three metal-containing structures were found with 3-naphthyl or 3-anthracenyl substituents. The interplanar angles for acetylacetonate $v s$ aryl fragments are $86.4^{\circ}$ for [3(1'-naphthyl)pentane-2,4-dionato][tris(2-aminoethyl)amine]cobalt(III) bis(tetrafluoroborate) dihydrate, $87.1^{\circ}$ for $\left[3-\left(2^{\prime}, 4^{\prime}-\right.\right.$ dinitro-1'-naphthyl)pentane-2,4-dionato][tris(2-aminoethyl)amine]cobalt(III) dibromide (BEYTEE and BIMLUE, respectively; Nakano \& Sato, 1982$)$ and $83.5^{\circ}$ for [3-(9'-anthryl)acetylacetonato]chlorido(1,4,7-trimethyl-1,4,7-triazacyclononane)iron(III) perchlorate mesitylene solvate (NUCZUG; Müller et al., 1998). These angles are in the same range as for the title compound.

\section{Synthesis and crystallization}

The starting $\mathrm{Tp}_{2} \mathrm{EuCl}$ complex was obtained by reaction of TpTl with $\mathrm{EuCl}_{3} \cdot 6 \mathrm{H}_{2} \mathrm{O}$ in methanol (Kandel et al., 2017). Then, $307 \mathrm{mg} \quad(0.50 \mathrm{mmol})$ of $\mathrm{Tp}_{2} \mathrm{EuCl}$ and $138 \mathrm{mg}$ $(0.50 \mathrm{mmol})$ of HAnthracac were dissolved in $15 \mathrm{~mL}$ of methylene chloride, followed by the addition of $0.15 \mathrm{~mL}$ of triethylamine. After the solution had been stirred for $1 \mathrm{~h}$, the reaction mixture was filtered and the filtrate was evaporated under reduced pressure (rotavapor). The resulting residue was washed with water and dried in a vacuum desiccator over $\mathrm{P}_{2} \mathrm{O}_{5}$. The crude product was recrystallized by slow diffusion of methyl $t$-butyl ether into a DMF solution of the compound. The title compound was obtained as orange prismatic crystals (25 mg, yield $10 \%$ ).

\section{Refinement}

Crystal data, data collection and structure refinement details are summarized in Table 3. $\mathrm{C}-\mathrm{H}$ bond distances were constrained to $0.95 \AA$ for aromatic and alkene $\mathrm{C}-\mathrm{H}$ moieties, and to $0.98 \AA$ for $\mathrm{CH}_{3}$ moieties. The $\mathrm{B}-\mathrm{H}$ bond distance was constrained to $1.00 \AA \AA$. $U_{\text {iso }}(\mathrm{H})$ values were set to $k U_{\text {eq }}(\mathrm{C})$ where $k=1.5$ for $\mathrm{CH}_{3}$ and 1.2 for $\mathrm{C}-\mathrm{H}$ units. 


\section{Funding information}

This work was partially supported by a grant from the National Research Foundation of Ukraine (project 2020.02/ 0202), and by the Target Program of Fundamental Research 'Prospective fundamental studies and innovative developments of nanomaterials and nanotechnologies for the needs of industry, health protection and agriculture', National Academy of Sciences of Ukraine (contracts No. 32/20-N and 32/21-N). We acknowledge the NSF-MRI program (grant No. CHE-1039027 to JPJ) for funding of the Gemini X-ray diffractometer.

\section{References}

Alvarez, S., Alemany, P., Casanova, D., Cirera, J., Llunell, M. \& Avnir, D. (2005). Coord. Chem. Rev. 249, 1693-1708.

Aulsebrook, M. L., Graham, B., Grace, M. R. \& Tuck, K. L. (2018). Coord. Chem. Rev. 375, 191-220.

Bortoluzzi, M., Paolucci, G., Gatto, M., Roppa, S., Enrichi, F., Ciorba, S. \& Richards, B. S. (2012). J. Lumin. 132, 2378-2384.

Bünzli, J.-C. G. (2017). Eur. J. Inorg. Chem. pp. 5058-5063.

Bünzli, J.-C. G. (2019). Trends Chem. 1, 751-762.

Carneiro Neto, A. N., Teotonio, E. E. S., de Sá, G. F., Brito, H. F., Legendziewicz, J., Carlos, L. D., Felinto, M. C. F. C., Gawryszewska, P., Moura, R. T. Jr, Longo, R. L., Faustino, W. M. \& Malta, O. L. (2019). Handbook on the Physics and Chemistry of Rare Earths, vol. 56, pp. 55-162. Amsterdam: Elsevier.

Casanova, D., Llunell, M., Alemany, P. \& Alvarez, S. (2005). Chem. Eur. J. 11, 1479-1494.

Davies, G. M., Adams, H. \& Ward, M. D. (2005). Acta Cryst. C61, $\mathrm{m} 221-\mathrm{m} 223$.

Dei, A., Gatteschi, D., Massa, C. A., Pardi, L. A., Poussereau, S. \& Sorace, L. (2000). Chem. Eur. J. 6, 4580-4586.

Dolomanov, O. V., Bourhis, L. J., Gildea, R. J., Howard, J. A. K. \& Puschmann, H. (2009). J. Appl. Cryst. 42, 339-341.

Galler, J. L., Goodchild, S., Gould, J., McDonald, R. \& Sella, A. (2004). Polyhedron, 23, 253-262.

Gheno, G., Bortoluzzi, M., Ganzerla, R. \& Enrichi, F. (2014). J. Lumin. 145, 963-969.

Groom, C. R., Bruno, I. J., Lightfoot, M. P. \& Ward, S. C. (2016). Acta Cryst. B72, 171-179.

Guégan, F., Riobé, F., Maury, O., Jung, J., Le Guennic, B., Morell, C. \& Luneau, D. (2018). Inorg. Chem. Front. 5, 1346-1353.

Kandel, A. V., Mikhalyova, E. A., Zeller, M., Addison, A. W. \& Pavlishchuk, V. V. (2017). Theor. Exp. Chem. 53, 180-186.

Khullar, S., Singh, S., Das, P. \& Mandal, S. K. (2019). ACS Omega, 4, 5283-5292.

Lawrence, R. G., Hamor, T. A., Jones, C. J., Paxton, K. \& Rowley, N. M. (2001). J. Chem. Soc. Dalton Trans. pp. 2121-2126.
Lawrence, R. G., Jones, C. J. \& Kresinski, R. A. (1996). J. Chem. Soc. Dalton Trans. pp. 501-507.

Lippard, S. L. \& Russ, B. I. (1968). Inorg. Chem. 7, 1686-1688.

Macrae, C. F., Sovago, I., Cottrell, S. J., Galek, P. T. A., McCabe, P., Pidcock, E., Platings, M., Shields, G. P., Stevens, J. S., Towler, M. \& Wood, P. A. (2020). J. Appl. Cryst. 53, 226-235.

Mikhalyova, E. A., Yakovenko, A. V., Zeller, M., Gavrilenko, K. S., Kiskin, M. A., Smola, S. S., Dotsenko, V. P., Eremenko, I. L., Addison, A. W. \& Pavlishchuk, V. V. (2017). Dalton Trans. 46, 3457-3469.

Mikhalyova, E. A., Zeller, M., Jasinski, J. P., Butcher, R. J., Carrella, L. M., Sedykh, A. E., Gavrilenko, K. S., Smola, S. S., Frasso, M., Cazorla, S. C., Perera, K., Shi, A., Ranjbar, H. G., Smith, C., Deac, A., Liu, Y., McGee, S. M., Dotsenko, V. P., Kumke, M. U., MüllerBuschbaum, K., Rentschler, E., Addison, A. W. \& Pavlishchuk, V. V. (2020). Dalton Trans. 49, 7774-7789.

Morissette, M., Haufe, S., McDonald, R., Ferrence, G. M. \& Takats, J. (2004). Polyhedron, 23, 263-271.

Moss, M. A. J., Jones, C. J. \& Edwards, A. J. (1988). Polyhedron, 7, 7981

Moss, M. A. J., Jones, C. J. \& Edwards, A. J. (1989). J. Chem. Soc. Dalton Trans. pp. 1393-1400.

Müller, M., Weyhermüller, T., Bill, E. \& Wieghardt, K. (1998). J. Biol. Inorg. Chem. 3, 96-106.

Nakano, Y. \& Sato, S. (1982). Inorg. Chem. 21, 1315-1318.

Nehra, K., Dalal, A., Hooda, A., Bhagwan, S., Saini, R. K., Mari, B., Kumar, S. \& Singh, D. (2022). J. Mol. Struct. 1249, 131531.

Onishi, M., Kayano, K., Inada, K., Yamaguchi, H., Nagaoka, J., Arikawa, Y. \& Takatani, T. (2004). Inorg. Chim. Acta, 357, 40914101.

Onishi, M., Yamaguchi, H., Shimotsuma, H., Hiraki, K., Nagaoka, J. \& Kawano, H. (1999). Chem. Lett. 28, 573-574.

Porai-Koshits, M. A. \& Aslanov, L. A. (1972). J. Struct. Chem. 13, 244-253.

Rigaku OD (2015). CrysAlisPro. Rigaku Oxford Diffraction, Yarnton, England.

Sarkar, D., Ganguli, S., Samanta, T. \& Mahalingam, V. (2019). Langmuir, 35, 6211-6230.

Sheldrick, G. M. (2015a). Acta Cryst. A71, 3-8.

Sheldrick, G. M. (2015b). Acta Cryst. C71, 3-8.

Venturini Filho, E., de Sousa Filho, P. C., Serra, O. A., Weber, I. T., Lucena, M. A. M. \& Luz, P. P. (2018). J. Lumin. 202, 89-96.

Wang, F., Pu, Y., Zhang, X., Zhang, F., Cheng, H. \& Zhao, Y. (2019). J. Lumin. 206, 192-198.

Wang, L., Zhao, Z., Wei, C., Wei, H., Liu, Z., Bian, Z. \& Huang, C. (2019). Adv. Opt. Mater. 7, 1801256.

Westrip, S. P. (2010). J. Appl. Cryst. 43, 920-925.

Zhang, H., Chen, Z.-H., Liu, X. \& Zhang, F. (2020). Nano Res. 13, 1795-1809. 


\section{supporting information}

Acta Cryst. (2022). E78, 103-107 [https://doi.org/10.1107/S2056989022000676]

Bis[3-(anthracen-9-yl) pentane-2,4-dionato- $\left.\kappa^{2} O, O^{\prime}\right](N, N$-dimethylformamide$\kappa O)\left[\right.$ tris(pyrazol-1-yl- $\kappa \mathrm{N}^{2}$ )hydroborato] europium(III)

Elena A. Mikhalyova, Matthias Zeller, Jerry P. Jasinski, Manpreet Kaur and Anthony W.

Addison

Computing details

Data collection: CrysAlis PRO (Rigaku OD, 2015); cell refinement: CrysAlis PRO (Rigaku OD, 2015); data reduction: CrysAlis PRO (Rigaku OD, 2015); program(s) used to solve structure: ShelXT (Sheldrick, 2015a); program(s) used to refine structure: SHELXL (Sheldrick, 2015b); molecular graphics: OLEX2 (Dolomanov et al., 2009), Mercury (Macrae et al., 2020); software used to prepare material for publication: OLEX2 (Dolomanov et al., 2009), and publCIF (Westrip, 2010).

Bis[3-(anthracen-9-yl) pentane-2,4-dionato- $\left.\kappa^{2} O, O^{\prime}\right](N, N$-dimethylformamide- $\kappa O)$ [tris (pyrazol-1-yl$\left.\kappa \mathrm{N}^{2}\right)$ hydroborato] europium(III)

Crystal data

$\left[\mathrm{Eu}\left(\mathrm{C}_{9} \mathrm{H}_{10} \mathrm{BN}_{6}\right)\left(\mathrm{C}_{19} \mathrm{H}_{15} \mathrm{O}_{2}\right)_{2}\left(\mathrm{C}_{3} \mathrm{H}_{7} \mathrm{NO}\right)\right]$

$M_{r}=988.71$

Monoclinic, $P 2{ }_{1} / n$

$a=9.3728(3) \AA$

$b=22.5555$ (7) $\AA$

$c=22.0840(6) \AA$

$\beta=96.314(3)^{\circ}$

$V=4640.4(2) \AA^{3}$

$Z=4$

$F(000)=2016$

$D_{\mathrm{x}}=1.415 \mathrm{Mg} \mathrm{m}^{-3}$

$\mathrm{Cu} K \alpha$ radiation, $\lambda=1.54184 \AA$

Cell parameters from 8094 reflections

$\theta=4.0-71.4^{\circ}$

$\mu=10.11 \mathrm{~mm}^{-1}$

$T=173 \mathrm{~K}$

Prism, orange

$0.48 \times 0.18 \times 0.12 \mathrm{~mm}$

Data collection

Rigaku Oxford Diffraction Gemini Eos diffractometer

Radiation source: fine-focus sealed X-ray tube, Enhance $(\mathrm{Cu})$ X-ray Source

Graphite monochromator

Detector resolution: 16.0416 pixels $\mathrm{mm}^{-1}$

$\omega$ scans

Absorption correction: multi-scan

(CrysAlisPro; Rigaku OD, 2015)

$T_{\min }=0.163, T_{\max }=1.000$

19671 measured reflections

8850 independent reflections

7261 reflections with $I>2 \sigma(I)$

$R_{\text {int }}=0.048$

$\theta_{\max }=71.4^{\circ}, \theta_{\min }=3.9^{\circ}$

$h=-11 \rightarrow 11$

$k=-27 \rightarrow 17$

$l=-26 \rightarrow 24$

Refinement

Refinement on $F^{2}$

Least-squares matrix: full

$R\left[F^{2}>2 \sigma\left(F^{2}\right)\right]=0.042$

$S=1.03$

8850 reflections

$w R\left(F^{2}\right)=0.106$

583 parameters

0 restraints 
Primary atom site location: dual

Hydrogen site location: inferred from neighbouring sites

$\mathrm{H}$-atom parameters constrained

$$
\begin{gathered}
w=1 /\left[\sigma^{2}\left(F_{\mathrm{o}}^{2}\right)+(0.0592 P)^{2}\right] \\
\text { where } P=\left(F_{\mathrm{o}}^{2}+2 F_{\mathrm{c}}{ }^{2}\right) / 3 \\
(\Delta / \sigma)_{\max }=0.004 \\
\Delta \rho_{\max }=1.09 \mathrm{e} \AA^{-3} \\
\Delta \rho_{\min }=-1.25 \mathrm{e} \AA^{-3}
\end{gathered}
$$

Special details

Geometry. All esds (except the esd in the dihedral angle between two 1.s. planes) are estimated using the full covariance

\begin{tabular}{|c|c|c|c|c|}
\hline & $x$ & $y$ & $z$ & $U_{\text {iso }} * / U_{\text {eq }}$ \\
\hline Eu1 & $0.52702(2)$ & $0.81156(2)$ & $0.43393(2)$ & $0.01770(8)$ \\
\hline $\mathrm{O} 1$ & 0.4093 & $0.77371(12)$ & $0.51430(12)$ & $0.0316(6)$ \\
\hline $\mathrm{O} 2$ & $0.4941(4)$ & $0.88721(12)$ & $0.50175(13)$ & $0.0396(8)$ \\
\hline $\mathrm{O} 3$ & $0.5692(3)$ & $0.70993(11)$ & $0.42273(11)$ & $0.0263(6)$ \\
\hline $\mathrm{O} 4$ & 0.7264 & 0.78408 (11) & $0.50130(13)$ & $0.0317(6)$ \\
\hline $\mathrm{O} 5$ & 0.2953 & $0.77901(14)$ & $0.38675(14)$ & $0.0383(7)$ \\
\hline N1 & 0.5855 (4) & $0.79579(14)$ & $0.32351(13)$ & $0.0248(7)$ \\
\hline N2 & $0.6057(4)$ & $0.83970(15)$ & $0.28333(14)$ & $0.0294(7)$ \\
\hline N3 & $0.7242(4)$ & $0.88341(14)$ & $0.41029(14)$ & $0.0266(7)$ \\
\hline N4 & $0.7259(4)$ & 0.91415 (14) & $0.35743(14)$ & $0.0268(7)$ \\
\hline N5 & $0.4131(4)$ & $0.89911(15)$ & $0.37142(16)$ & $0.0331(8)$ \\
\hline N6 & $0.4623(4)$ & $0.92238(15)$ & $0.32156(16)$ & $0.0361(8)$ \\
\hline N7 & $0.0779(5)$ & $0.7911(2)$ & $0.3344(3)$ & $0.0597(14)$ \\
\hline $\mathrm{C} 1$ & $0.3798(4)$ & $0.79077(17)$ & $0.56538(18)$ & $0.0266(8)$ \\
\hline $\mathrm{C} 2$ & $0.4045(5)$ & $0.84859(17)$ & $0.58988(17)$ & $0.0269(8)$ \\
\hline $\mathrm{C} 3$ & $0.4583(5)$ & $0.89398(18)$ & 0.55457 (19) & $0.0339(10)$ \\
\hline $\mathrm{C} 4$ & $0.3751(5)$ & $0.86189(16)$ & $0.65368(18)$ & $0.0298(9)$ \\
\hline $\mathrm{C} 5$ & $0.2383(5)$ & $0.88156(16)$ & $0.6657(2)$ & $0.0345(10)$ \\
\hline C6 & $0.1212(5)$ & $0.88699(18)$ & $0.6203(2)$ & $0.0424(11)$ \\
\hline H6 & 0.1357 & 0.8791 & 0.5792 & $0.051 *$ \\
\hline $\mathrm{C} 7$ & $-0.0128(6)$ & $0.9033(2)$ & 0.6333 & $0.0591(16)$ \\
\hline $\mathrm{H} 7$ & -0.0900 & 0.9063 & 0.6018 & $0.071 *$ \\
\hline $\mathrm{C} 8$ & $-0.0347(9)$ & $0.9156(2)$ & $0.6950(4)$ & $0.079(3)$ \\
\hline H8 & -0.1274 & 0.9266 & 0.7046 & $0.094 *$ \\
\hline $\mathrm{C} 9$ & $0.0737(9)$ & $0.9117(2)$ & 0.7394 & $0.071(2)$ \\
\hline H9 & 0.0562 & 0.9201 & 0.7800 & $0.085^{*}$ \\
\hline $\mathrm{C} 10$ & $0.2147(7)$ & $0.89522(18)$ & $0.7277(2)$ & $0.0496(15)$ \\
\hline C11 & $0.3259(8)$ & $0.8903(2)$ & $0.7737(2)$ & $0.0584(18)$ \\
\hline H11 & 0.3095 & 0.9007 & 0.8140 & $0.070 *$ \\
\hline $\mathrm{C} 12$ & $0.4595(7)$ & $0.87091(19)$ & $0.7630(2)$ & $0.0501(14)$ \\
\hline C13 & $0.5756(10)$ & $0.8643(2)$ & $0.8103(3)$ & $0.072(2)$ \\
\hline H13 & 0.5615 & 0.8757 & 0.8506 & $0.086^{*}$ \\
\hline $\mathrm{C} 14$ & $0.7041(10)$ & $0.8424(3)$ & $0.7996(3)$ & $0.084(3)$ \\
\hline H14 & 0.7790 & 0.8385 & 0.8320 & $0.101 *$ \\
\hline
\end{tabular}
matrix. The cell esds are taken into account individually in the estimation of esds in distances, angles and torsion angles; correlations between esds in cell parameters are only used when they are defined by crystal symmetry. An approximate (isotropic) treatment of cell esds is used for estimating esds involving l.s. planes.

Fractional atomic coordinates and isotropic or equivalent isotropic displacement parameters $\left(\AA^{2}\right)$ 


\begin{tabular}{|c|c|c|c|c|}
\hline $\mathrm{C} 15$ & $0.7271(7)$ & $0.8252(3)$ & $0.7400(3)$ & $0.0683(19)$ \\
\hline H15 & 0.8169 & 0.8088 & 0.7328 & $0.082 *$ \\
\hline $\mathrm{C} 16$ & $0.6219(6)$ & $0.8320(2)$ & $0.6926(2)$ & $0.0471(12)$ \\
\hline H16 & 0.6406 & 0.8211 & 0.6527 & $0.056^{*}$ \\
\hline $\mathrm{C} 17$ & $0.4842(5)$ & $0.85520(18)$ & $0.70201(19)$ & $0.0360(10)$ \\
\hline $\mathrm{C} 18$ & $0.6316(5)$ & $0.66956(16)$ & $0.45555(16)$ & $0.0250(8)$ \\
\hline C19 & $0.7273(4)$ & $0.67949(16)$ & $0.50837(16)$ & $0.0253(8)$ \\
\hline $\mathrm{C} 20$ & $0.7690(4)$ & $0.73674(18)$ & $0.52778(17)$ & $0.0271(8)$ \\
\hline $\mathrm{C} 21$ & $0.7840(5)$ & $0.62631(17)$ & $0.54511(18)$ & $0.0353(10)$ \\
\hline $\mathrm{C} 22$ & $0.6957(6)$ & $0.59931(18)$ & $0.58541(19)$ & $0.0417(12)$ \\
\hline $\mathrm{C} 23$ & $0.5594(6)$ & $0.6217(2)$ & $0.5950(2)$ & $0.0448(12)$ \\
\hline $\mathrm{H} 23$ & 0.5257 & 0.6567 & 0.5741 & $0.054^{*}$ \\
\hline $\mathrm{C} 24$ & $0.4744(7)$ & $0.5949(3)$ & $0.6332(2)$ & $0.0580(15)$ \\
\hline $\mathrm{H} 24$ & 0.3843 & 0.6118 & 0.6394 & $0.070^{*}$ \\
\hline $\mathrm{C} 25$ & $0.5201(10)$ & $0.5420(3)$ & $0.6635(3)$ & 0.075 \\
\hline $\mathrm{H} 25$ & 0.4595 & 0.5229 & 0.6892 & $0.090 *$ \\
\hline $\mathrm{C} 26$ & $0.6484(9)$ & $0.5188(2)$ & $0.6562(2)$ & $0.0654(19)$ \\
\hline $\mathrm{H} 26$ & 0.6768 & 0.4828 & 0.6765 & $0.079 *$ \\
\hline $\mathrm{C} 27$ & $0.7442(7)$ & $0.5466(2)$ & $0.6185(2)$ & $0.0503(14)$ \\
\hline $\mathrm{C} 28$ & $0.8772(7)$ & $0.5250(2)$ & $0.6124(2)$ & $0.0587(17)$ \\
\hline $\mathrm{H} 28$ & 0.9088 & 0.4905 & 0.6347 & $0.070^{*}$ \\
\hline $\mathrm{C} 29$ & $0.9690(7)$ & $0.5512(2)$ & $0.5747(2)$ & $0.0540(15)$ \\
\hline C30 & $1.1070(8)$ & 0.5289 & $0.5678(3)$ & $0.074(2)$ \\
\hline $\mathrm{H} 30$ & 1.1411 & 0.4948 & 0.5903 & $0.089 *$ \\
\hline $\mathrm{C} 31$ & $1.1914(8)$ & $0.5550(3)$ & $0.5299(3)$ & $0.071(2)$ \\
\hline H31 & 1.2842 & 0.5391 & 0.5269 & $0.085^{*}$ \\
\hline C32 & $1.1462(7)$ & $0.6054(3)$ & $0.4942(3)$ & $0.0692(19)$ \\
\hline H32 & 1.2067 & 0.6229 & 0.4674 & $0.083^{*}$ \\
\hline $\mathrm{C} 33$ & $1.0122(6)$ & $0.6281(2)$ & $0.4999(2)$ & $0.0511(13)$ \\
\hline H33 & 0.9806 & 0.6620 & 0.4764 & $0.061 *$ \\
\hline C34 & $0.9201(6)$ & $0.6030(2)$ & $0.5391(2)$ & $0.0434(12)$ \\
\hline $\mathrm{C} 35$ & $0.2815(6)$ & $0.9201(2)$ & $0.3731(3)$ & $0.0520(13)$ \\
\hline H35 & 0.2225 & 0.9118 & 0.4043 & $0.062 *$ \\
\hline C36 & $0.2421(8)$ & $0.9556(3)$ & $0.3235(4)$ & $0.076(2)$ \\
\hline H36 & 0.1529 & 0.9753 & 0.3135 & $0.091 *$ \\
\hline $\mathrm{C} 37$ & $0.3605(7)$ & $0.9565(3)$ & $0.2915(3)$ & $0.0662(17)$ \\
\hline H37 & 0.3686 & 0.9774 & 0.2547 & $0.079^{*}$ \\
\hline C38 & $0.6116(6)$ & $0.8170(2)$ & $0.22766(18)$ & $0.0374(10)$ \\
\hline H38 & 0.6252 & 0.8389 & 0.1920 & $0.045^{*}$ \\
\hline C39 & $0.5948(5)$ & $0.7566(2)$ & $0.23078(18)$ & $0.0373(10)$ \\
\hline H39 & 0.5933 & 0.7287 & 0.1985 & $0.045^{*}$ \\
\hline $\mathrm{C} 40$ & $0.5805(5)$ & $0.74553(18)$ & $0.29162(17)$ & $0.0282(8)$ \\
\hline $\mathrm{H} 40$ & 0.5688 & 0.7072 & 0.3083 & $0.034 *$ \\
\hline $\mathrm{C} 41$ & $0.8332(5)$ & $0.9041(2)$ & $0.4480(2)$ & $0.0384(10)$ \\
\hline H41 & 0.8576 & 0.8906 & 0.4886 & $0.046^{*}$ \\
\hline $\mathrm{C} 42$ & $0.9064(6)$ & $0.9479(2)$ & $0.4203(2)$ & $0.0476(12)$ \\
\hline H42 & 0.9882 & 0.9695 & 0.4372 & $0.057^{*}$ \\
\hline $\mathrm{C} 43$ & $0.8344(6)$ & $0.95314(19)$ & $0.3629(2)$ & $0.0410(11)$ \\
\hline
\end{tabular}




$\begin{array}{lllll}\text { H43 } & 0.8576 & 0.9799 & 0.3323 & 0.049^{*} \\ \text { C44 } & 0.4746(8) & 0.9563(2) & 0.5808(2) & \left.0.067^{*}\right) \\ \text { H44A } & 0.5506 & 0.9772 & 0.5623 & 0.101^{*} \\ \text { H44B } & 0.4998 & 0.9540 & 0.6250 & 0.101^{*} \\ \text { H44C } & 0.3839 & 0.9778 & 0.5720 & 0.101^{*} \\ \text { C45 } & 0.3115(6) & 0.74539(18) & 0.6032(2) & 0.0405^{*}(1) \\ \text { H45A } & 0.2855 & 0.7102 & 0.5783 & 0.061^{*} \\ \text { H45B } & 0.2251 & 0.7623 & 0.6177 & 0.061^{*} \\ \text { H45C } & 0.3796 & 0.7342 & 0.6382 & 0.061^{*} \\ \text { C46 } & 0.8711(6) & 0.7442(2) & 0.5854(2) & 0.0445(12) \\ \text { H46A } & 0.9668 & 0.7302 & 0.5782 & 0.067^{*} \\ \text { H46B } & 0.8363 & 0.7211 & 0.6183 & 0.067^{*} \\ \text { H46C } & 0.8762 & 0.7862 & 0.5968 & 0.067^{*} \\ \text { C47 } & 0.5960(6) & 0.60688(18) & 0.43470(19) & 0.0394(11) \\ \text { H47A } & 0.5374 & 0.5879 & 0.4633 & 0.059^{*} \\ \text { H47B } & 0.6850 & 0.5844 & 0.4333 & 0.059^{*} \\ \text { H47C } & 0.5424 & 0.6078 & 0.3940 & 0.059^{*} \\ \text { C48 } & 0.2187(6) & 0.7920(2) & 0.3402(3) & 0.0481(12) \\ \text { H48 } & 0.2645 & 0.8035 & 0.3057 & 0.058^{*} \\ \text { C49 } & -0.0009(10) & 0.8083(3) & 0.2768(4) & 0.106(4) \\ \text { H49A } & 0.0668 & 0.8210 & 0.2485 & 0.158^{*} \\ \text { H49B } & -0.0658 & 0.8412 & 0.2835 & 0.158^{*} \\ \text { H49C } & -0.0568 & 0.7745 & 0.2594 & 0.158^{*} \\ \text { C50 } & -0.0025(8) & 0.7747(4) & 0.3832(4) & 0.094(3) \\ \text { H50A } & 0.0636 & 0.7644 & 0.4192 & 0.141^{*} \\ \text { H50B } & -0.0630 & 0.7404 & 0.3709 & 0.141^{*} \\ \text { H50C } & -0.0632 & 0.8079 & 0.3929 & 0.141^{*} \\ \text { B1 } & 0.6109(6) & 0.9057(2) & 0.3029(2) & 0.0331(11) \\ \text { H1 } & 0.6336 & 0.9311 & 0.2681 & 0.040^{*} \\ & & & & \end{array}$

Atomic displacement parameters $\left(\hat{A}^{2}\right)$

\begin{tabular}{lllllll}
\hline & $U^{11}$ & $U^{22}$ & $U^{33}$ & $U^{12}$ & $U^{13}$ & $U^{23}$ \\
\hline Eu1 & $0.02659(12)$ & $0.01614(10)$ & $0.01118(10)$ & $0.00364(9)$ & $0.00566(7)$ & $-0.00032(7)$ \\
O1 & $0.0516(18)$ & $0.0232(13)$ & $0.0240(13)$ & $-0.0069(12)$ & $0.0212(13)$ & $-0.0064(10)$ \\
O2 & $0.079(2)$ & $0.0166(12)$ & $0.0292(14)$ & $-0.0018(14)$ & $0.0342(15)$ & $-0.0044(10)$ \\
O3 & $0.0391(15)$ & $0.0230(12)$ & $0.0160(11)$ & $0.0072(11)$ & $-0.0003(11)$ & $0.0018(10)$ \\
O4 & $0.0422(17)$ & $0.0186(12)$ & $0.0318(14)$ & $-0.0022(12)$ & $-0.0072(13)$ & $0.0049(11)$ \\
O5 & $0.0329(16)$ & $0.0390(16)$ & $0.0406(17)$ & $-0.0004(13)$ & $-0.0067(14)$ & $0.0018(13)$ \\
N1 & $0.0393(19)$ & $0.0235(15)$ & $0.0127(13)$ & $0.0060(13)$ & $0.0075(13)$ & $-0.0013(11)$ \\
N2 & $0.046(2)$ & $0.0281(17)$ & $0.0149(14)$ & $0.0025(15)$ & $0.0055(14)$ & $0.0022(12)$ \\
N3 & $0.0397(19)$ & $0.0229(14)$ & $0.0178(14)$ & $-0.0003(14)$ & $0.0060(14)$ & $0.0029(12)$ \\
N4 & $0.0397(19)$ & $0.0201(14)$ & $0.0225(15)$ & $-0.0010(14)$ & $0.0123(14)$ & $-0.0017(12)$ \\
N5 & $0.037(2)$ & $0.0259(16)$ & $0.0366(19)$ & $0.0130(15)$ & $0.0052(16)$ & $0.0034(14)$ \\
N6 & $0.050(2)$ & $0.0284(17)$ & $0.0277(17)$ & $0.0182(16)$ & $-0.0042(16)$ & $0.0058(13)$ \\
N7 & $0.039(2)$ & $0.041(2)$ & $0.094(4)$ & $0.0157(19)$ & $-0.021(3)$ & $-0.021(2)$ \\
C1 & $0.033(2)$ & $0.0217(17)$ & $0.0267(18)$ & $-0.0019(15)$ & $0.0119(16)$ & $-0.0023(15)$ \\
C2 & $0.040(2)$ & $0.0227(17)$ & $0.0206(17)$ & $0.0004(16)$ & $0.0159(16)$ & $-0.0031(14)$
\end{tabular}




\begin{tabular}{|c|c|c|c|c|c|c|}
\hline $\mathrm{C} 3$ & $0.056(3)$ & $0.0205(18)$ & $0.028(2)$ & $-0.0003(18)$ & $0.019(2)$ & $-0.0025(15)$ \\
\hline $\mathrm{C} 4$ & $0.048(2)$ & $0.0183(17)$ & $0.0268(19)$ & $-0.0071(17)$ & $0.0188(18)$ & -0.0059 (14) \\
\hline $\mathrm{C} 5$ & $0.054(3)$ & $0.0143(16)$ & $0.040(2)$ & $-0.0052(17)$ & $0.028(2)$ & $-0.0038(15)$ \\
\hline C6 & $0.047(3)$ & $0.0206(18)$ & $0.064(3)$ & $-0.0014(19)$ & $0.027(2)$ & -0.0067 (19) \\
\hline $\mathrm{C} 7$ & 0.050 & $0.024(2)$ & $0.108(5)$ & $0.000(2)$ & $0.028(3)$ & $-0.001(3)$ \\
\hline $\mathrm{C} 8$ & $0.092(5)$ & $0.022(2)$ & $0.140(7)$ & 0.009 & $0.090(5)$ & $0.010(3)$ \\
\hline $\mathrm{C} 9$ & $0.115(6)$ & $0.019(2)$ & $0.093(5)$ & $0.007(3)$ & $0.085(5)$ & $0.000(3)$ \\
\hline $\mathrm{C} 10$ & $0.091(4)$ & $0.0167(18)$ & $0.051(3)$ & $-0.005(2)$ & $0.052(3)$ & $-0.0055(17)$ \\
\hline C11 & $0.127(6)$ & $0.028(2)$ & $0.027(2)$ & $-0.016(3)$ & $0.040(3)$ & $-0.0104(17)$ \\
\hline $\mathrm{C} 12$ & $0.101(5)$ & $0.023(2)$ & $0.028(2)$ & $-0.017(2)$ & $0.013(3)$ & $-0.0027(16)$ \\
\hline $\mathrm{C} 13$ & $0.146(7)$ & $0.031(3)$ & $0.034(3)$ & $-0.028(4)$ & $-0.007(4)$ & $0.002(2)$ \\
\hline $\mathrm{C} 14$ & $0.126(7)$ & $0.053(4)$ & $0.062(4)$ & $-0.045(4)$ & $-0.045(5)$ & $0.017(3)$ \\
\hline C15 & $0.063(4)$ & $0.057(3)$ & $0.079(4)$ & $-0.022(3)$ & $-0.015(3)$ & $0.023(3)$ \\
\hline $\mathrm{C} 16$ & 0.049 & $0.045(3)$ & $0.047(3)$ & $-0.016(2)$ & $0.006(2)$ & $0.005(2)$ \\
\hline $\mathrm{C} 17$ & $0.059(3)$ & $0.0224(18)$ & $0.028(2)$ & -0.0147 (19) & $0.011(2)$ & $0.0014(15)$ \\
\hline $\mathrm{C} 18$ & 0.039 (2) & $0.0197(17)$ & $0.0168(16)$ & $0.0072(16)$ & $0.0052(15)$ & $-0.0013(13)$ \\
\hline C19 & $0.035(2)$ & $0.0214(17)$ & $0.0191(16)$ & $0.0052(15)$ & $0.0000(15)$ & 0.0017 (13) \\
\hline $\mathrm{C} 20$ & $0.029(2)$ & $0.0293(19)$ & $0.0221(17)$ & $0.0038(16)$ & $-0.0012(16)$ & $0.0035(15)$ \\
\hline $\mathrm{C} 21$ & $0.054(3)$ & $0.0222(18)$ & $0.0261(19)$ & $0.0072(18)$ & $-0.0128(19)$ & $-0.0028(15)$ \\
\hline $\mathrm{C} 22$ & $0.075(4)$ & $0.0229(19)$ & $0.0226(19)$ & $-0.002(2)$ & $-0.012(2)$ & $0.0012(15)$ \\
\hline $\mathrm{C} 23$ & $0.066(3)$ & $0.035(2)$ & $0.033(2)$ & $-0.005(2)$ & $-0.001(2)$ & $0.0079(18)$ \\
\hline $\mathrm{C} 24$ & $0.080(4)$ & $0.052(3)$ & $0.042(3)$ & $-0.017(3)$ & 0.009 (3) & $0.010(2)$ \\
\hline $\mathrm{C} 25$ & $0.114(6)$ & 0.059 (4) & $0.050(3)$ & $-0.029(4)$ & $0.001(4)$ & $0.022(3)$ \\
\hline $\mathrm{C} 26$ & $0.115(6)$ & $0.034(3)$ & $0.043(3)$ & $-0.016(3)$ & $-0.012(3)$ & $0.019(2)$ \\
\hline $\mathrm{C} 27$ & 0.087 (4) & $0.025(2)$ & $0.034(2)$ & $0.004(2)$ & $-0.018(3)$ & 0.0059 (18) \\
\hline $\mathrm{C} 28$ & $0.102(5)$ & $0.029(2)$ & $0.040(3)$ & $0.015(3)$ & $-0.017(3)$ & $0.003(2)$ \\
\hline $\mathrm{C} 29$ & $0.070(4)$ & $0.041(3)$ & $0.044(3)$ & $0.024(3)$ & $-0.026(3)$ & $-0.017(2)$ \\
\hline $\mathrm{C} 30$ & $0.090(5)$ & $0.055(3)$ & $0.068(4)$ & $0.038(4)$ & $-0.033(4)$ & $-0.012(3)$ \\
\hline $\mathrm{C} 31$ & $0.066(4)$ & $0.071(4)$ & $0.069(4)$ & $0.040(4)$ & $-0.023(3)$ & $-0.019(3)$ \\
\hline $\mathrm{C} 32$ & $0.057(4)$ & $0.089(5)$ & $0.058(4)$ & $0.017(3)$ & -0.010 & $-0.028(3)$ \\
\hline $\mathrm{C} 33$ & $0.051(3)$ & $0.048(3)$ & $0.050(3)$ & $0.020(2)$ & $-0.011(2)$ & $-0.013(2)$ \\
\hline $\mathrm{C} 34$ & $0.056(3)$ & $0.034(2)$ & $0.036(2)$ & $0.014(2)$ & $-0.014(2)$ & $-0.0124(18)$ \\
\hline $\mathrm{C} 35$ & $0.044(3)$ & $0.032(2)$ & $0.080(4)$ & $0.022(2)$ & 0.007 (3) & $0.005(2)$ \\
\hline $\mathrm{C} 36$ & $0.061(4)$ & $0.056(4)$ & $0.109(6)$ & $0.033(3)$ & $0.000(4)$ & $0.020(4)$ \\
\hline $\mathrm{C} 37$ & $0.076(4)$ & $0.051(3)$ & $0.068(4)$ & $0.023(3)$ & $-0.007(3)$ & $0.024(3)$ \\
\hline $\mathrm{C} 38$ & $0.056(3)$ & $0.042(2)$ & $0.0161(17)$ & $-0.004(2)$ & $0.0130(18)$ & $0.0007(16)$ \\
\hline C39 & $0.056(3)$ & $0.039(2)$ & $0.0193(18)$ & $-0.003(2)$ & $0.0113(19)$ & $-0.0079(16)$ \\
\hline $\mathrm{C} 40$ & $0.037(2)$ & $0.0282(19)$ & $0.0200(18)$ & $0.0001(17)$ & $0.0070(16)$ & $-0.0047(14)$ \\
\hline $\mathrm{C} 41$ & $0.044(3)$ & $0.036(2)$ & $0.034(2)$ & $-0.006(2)$ & $-0.002(2)$ & $-0.0020(17)$ \\
\hline $\mathrm{C} 42$ & $0.051(3)$ & $0.036(2)$ & $0.056(3)$ & $-0.014(2)$ & $0.005(2)$ & $-0.004(2)$ \\
\hline $\mathrm{C} 43$ & 0.057 (3) & $0.027(2)$ & $0.042(2)$ & $-0.009(2)$ & $0.021(2)$ & -0.0025 (17) \\
\hline $\mathrm{C} 44$ & $0.140(6)$ & $0.023(2)$ & 0.049 (3) & $-0.020(3)$ & $0.060(4)$ & $-0.012(2)$ \\
\hline $\mathrm{C} 45$ & $0.062(3)$ & $0.0257(19)$ & $0.040(2)$ & $-0.014(2)$ & $0.032(2)$ & -0.0065 (17) \\
\hline $\mathrm{C} 46$ & $0.054(3)$ & $0.031(2)$ & $0.042(3)$ & $0.002(2)$ & $-0.023(2)$ & $-0.0043(18)$ \\
\hline $\mathrm{C} 47$ & $0.064(3)$ & $0.0226(19)$ & $0.028(2)$ & $0.003(2)$ & $-0.011(2)$ & $-0.0035(15)$ \\
\hline $\mathrm{C} 48$ & $0.051(3)$ & $0.035(2)$ & $0.056(3)$ & $0.006(2)$ & $-0.007(2)$ & $-0.004(2)$ \\
\hline $\mathrm{C} 49$ & $0.100(6)$ & $0.077(5)$ & 0.125 & $0.032(5)$ & $-0.056(6)$ & $-0.029(5)$ \\
\hline $\mathrm{C} 50$ & 0.062 & $0.090(6)$ & $0.135(8)$ & -0.007 & $0.035(5)$ & $-0.044(5)$ \\
\hline
\end{tabular}




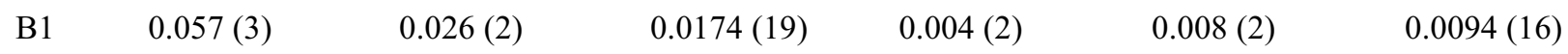

Geometric parameters $\left(\AA,{ }^{\circ}\right)$

\begin{tabular}{|c|c|c|c|}
\hline $\mathrm{Eu} 1-\mathrm{O} 1$ & $2.351(3)$ & $\mathrm{C} 20-\mathrm{C} 46$ & $1.515(5)$ \\
\hline $\mathrm{Eu} 1-\mathrm{O} 2$ & $2.313(3)$ & $\mathrm{C} 21-\mathrm{C} 22$ & $1.418(7)$ \\
\hline $\mathrm{Eu} 1-\mathrm{O} 3$ & $2.344(3)$ & $\mathrm{C} 21-\mathrm{C} 34$ & $1.399(7)$ \\
\hline $\mathrm{Eu} 1-\mathrm{O} 4$ & $2.340(3)$ & $\mathrm{C} 22-\mathrm{C} 23$ & $1.411(8)$ \\
\hline Eu1-O5 & $2.417(3)$ & $\mathrm{C} 22-\mathrm{C} 27$ & $1.443(6)$ \\
\hline $\mathrm{Eu} 1-\mathrm{N} 1$ & $2.582(3)$ & $\mathrm{C} 23-\mathrm{H} 23$ & 0.9500 \\
\hline $\mathrm{Eu} 1-\mathrm{N} 3$ & $2.555(3)$ & $\mathrm{C} 23-\mathrm{C} 24$ & $1.364(7)$ \\
\hline Eu1-N5 & $2.573(3)$ & $\mathrm{C} 24-\mathrm{H} 24$ & 0.9500 \\
\hline $\mathrm{O} 1-\mathrm{C} 1$ & $1.251(5)$ & $\mathrm{C} 24-\mathrm{C} 25$ & $1.410(9)$ \\
\hline $\mathrm{O} 2-\mathrm{C} 3$ & $1.258(5)$ & $\mathrm{C} 25-\mathrm{H} 25$ & 0.9500 \\
\hline $\mathrm{O} 3-\mathrm{C} 18$ & $1.266(5)$ & $\mathrm{C} 25-\mathrm{C} 26$ & $1.339(10)$ \\
\hline $\mathrm{O} 4-\mathrm{C} 20$ & $1.261(5)$ & $\mathrm{C} 26-\mathrm{H} 26$ & 0.9500 \\
\hline $\mathrm{O} 5-\mathrm{C} 48$ & $1.223(6)$ & $\mathrm{C} 26-\mathrm{C} 27$ & $1.434(9)$ \\
\hline $\mathrm{N} 1-\mathrm{N} 2$ & $1.357(5)$ & $\mathrm{C} 27-\mathrm{C} 28$ & $1.359(9)$ \\
\hline $\mathrm{N} 1-\mathrm{C} 40$ & $1.333(5)$ & $\mathrm{C} 28-\mathrm{H} 28$ & 0.9500 \\
\hline $\mathrm{N} 2-\mathrm{C} 38$ & 1.339 (5) & $\mathrm{C} 28-\mathrm{C} 29$ & $1.393(9)$ \\
\hline $\mathrm{N} 2-\mathrm{B} 1$ & $1.549(6)$ & $\mathrm{C} 29-\mathrm{C} 30$ & $1.411(9)$ \\
\hline $\mathrm{N} 3-\mathrm{N} 4$ & $1.359(4)$ & $\mathrm{C} 29-\mathrm{C} 34$ & $1.455(7)$ \\
\hline N3-C41 & $1.330(6)$ & $\mathrm{C} 30-\mathrm{H} 30$ & 0.9500 \\
\hline $\mathrm{N} 4-\mathrm{C} 43$ & $1.340(6)$ & $\mathrm{C} 30-\mathrm{C} 31$ & $1.348(11)$ \\
\hline $\mathrm{N} 4-\mathrm{B} 1$ & $1.538(6)$ & $\mathrm{C} 31-\mathrm{H} 31$ & 0.9500 \\
\hline N5-N6 & $1.346(5)$ & $\mathrm{C} 31-\mathrm{C} 32$ & $1.422(10)$ \\
\hline N5-C35 & $1.326(6)$ & C $32-\mathrm{H} 32$ & 0.9500 \\
\hline N6-C37 & $1.343(6)$ & $\mathrm{C} 32-\mathrm{C} 33$ & $1.374(8)$ \\
\hline N6-B1 & $1.542(7)$ & C $33-\mathrm{H} 33$ & 0.9500 \\
\hline N7-C48 & $1.312(7)$ & $\mathrm{C} 33-\mathrm{C} 34$ & $1.407(8)$ \\
\hline N7-C49 & $1.452(9)$ & C35-H35 & 0.9500 \\
\hline $\mathrm{N} 7-\mathrm{C} 50$ & $1.431(10)$ & $\mathrm{C} 35-\mathrm{C} 36$ & $1.374(9)$ \\
\hline $\mathrm{C} 1-\mathrm{C} 2$ & $1.421(5)$ & C36- & 0.9500 \\
\hline $\mathrm{C} 1-\mathrm{C} 45$ & $1.507(5)$ & $\mathrm{C} 36-\mathrm{C} 37$ & $1.380(10)$ \\
\hline $\mathrm{C} 2-\mathrm{C} 3$ & $1.413(5)$ & С $37-\mathrm{H} 37$ & 0.9500 \\
\hline $\mathrm{C} 2-\mathrm{C} 4$ & $1.495(5)$ & C $38-\mathrm{H} 38$ & 0.9500 \\
\hline $\mathrm{C} 3-\mathrm{C} 44$ & $1.522(6)$ & $\mathrm{C} 38-\mathrm{C} 39$ & $1.374(6)$ \\
\hline $\mathrm{C} 4-\mathrm{C} 5$ & $1.409(6)$ & C39-H39 & 0.9500 \\
\hline $\mathrm{C} 4-\mathrm{C} 17$ & $1.402(6)$ & $\mathrm{C} 39-\mathrm{C} 40$ & $1.387(5)$ \\
\hline $\mathrm{C} 5-\mathrm{C} 6$ & $1.408(7)$ & $\mathrm{C} 40-\mathrm{H} 40$ & 0.9500 \\
\hline $\mathrm{C} 5-\mathrm{C} 10$ & $1.445(6)$ & $\mathrm{C} 41-\mathrm{H} 41$ & 0.9500 \\
\hline C6-H6 & 0.9500 & $\mathrm{C} 41-\mathrm{C} 42$ & $1.383(7)$ \\
\hline $\mathrm{C} 6-\mathrm{C} 7$ & $1.370(7)$ & $\mathrm{C} 42-\mathrm{H} 42$ & 0.9500 \\
\hline $\mathrm{C} 7-\mathrm{H} 7$ & 0.9500 & $\mathrm{C} 42-\mathrm{C} 43$ & $1.374(7)$ \\
\hline $\mathrm{C} 7-\mathrm{C} 8$ & $1.427(10)$ & $\mathrm{C} 43-\mathrm{H} 43$ & 0.9500 \\
\hline $\mathrm{C} 8-\mathrm{H} 8$ & 0.9500 & $\mathrm{C} 44-\mathrm{H} 44 \mathrm{~A}$ & 0.9800 \\
\hline $\mathrm{C} 8-\mathrm{C} 9$ & $1.335(10)$ & $\mathrm{C} 44-\mathrm{H} 44 \mathrm{~B}$ & 0.9800 \\
\hline $\mathrm{C} 9-\mathrm{H} 9$ & 0.9500 & $\mathrm{C} 44-\mathrm{H} 44 \mathrm{C}$ & 0.9800 \\
\hline
\end{tabular}




\begin{tabular}{|c|c|c|c|}
\hline $\mathrm{C} 9-\mathrm{C} 10$ & $1.423(9)$ & $\mathrm{C} 45-\mathrm{H} 45 \mathrm{~A}$ & 0.9800 \\
\hline $\mathrm{C} 10-\mathrm{C} 11$ & $1.377(9)$ & $\mathrm{C} 45-\mathrm{H} 45 \mathrm{~B}$ & 0.9800 \\
\hline $\mathrm{C} 11-\mathrm{H} 11$ & 0.9500 & $\mathrm{C} 45-\mathrm{H} 45 \mathrm{C}$ & 0.9800 \\
\hline $\mathrm{C} 11-\mathrm{C} 12$ & $1.372(9)$ & $\mathrm{C} 46-\mathrm{H} 46 \mathrm{~A}$ & 0.9800 \\
\hline $\mathrm{C} 12-\mathrm{C} 13$ & $1.430(9)$ & $\mathrm{C} 46-\mathrm{H} 46 \mathrm{~B}$ & 0.9800 \\
\hline $\mathrm{C} 12-\mathrm{C} 17$ & $1.436(6)$ & $\mathrm{C} 46-\mathrm{H} 46 \mathrm{C}$ & 0.9800 \\
\hline C13-H13 & 0.9500 & $\mathrm{C} 47-\mathrm{H} 47 \mathrm{~A}$ & 0.9800 \\
\hline $\mathrm{C} 13-\mathrm{C} 14$ & $1.347(11)$ & C47-H47B & 0.9800 \\
\hline C14-H14 & 0.9500 & $\mathrm{C} 47-\mathrm{H} 47 \mathrm{C}$ & 0.9800 \\
\hline $\mathrm{C} 14-\mathrm{C} 15$ & $1.409(11)$ & $\mathrm{C} 48-\mathrm{H} 48$ & 0.9500 \\
\hline C15-H15 & 0.9500 & $\mathrm{C} 49-\mathrm{H} 49 \mathrm{~A}$ & 0.9800 \\
\hline $\mathrm{C} 15-\mathrm{C} 16$ & $1.366(8)$ & $\mathrm{C} 49-\mathrm{H} 49 \mathrm{~B}$ & 0.9800 \\
\hline C16-H16 & 0.9500 & $\mathrm{C} 49-\mathrm{H} 49 \mathrm{C}$ & 0.9800 \\
\hline $\mathrm{C} 16-\mathrm{C} 17$ & $1.429(7)$ & $\mathrm{C} 50-\mathrm{H} 50 \mathrm{~A}$ & 0.9800 \\
\hline $\mathrm{C} 18-\mathrm{C} 19$ & $1.409(5)$ & $\mathrm{C} 50-\mathrm{H} 50 \mathrm{~B}$ & 0.9800 \\
\hline $\mathrm{C} 18-\mathrm{C} 47$ & $1.513(5)$ & $\mathrm{C} 50-\mathrm{H} 50 \mathrm{C}$ & 0.9800 \\
\hline $\mathrm{C} 19-\mathrm{C} 20$ & $1.402(5)$ & $\mathrm{B} 1-\mathrm{H} 1$ & 1.0000 \\
\hline $\mathrm{C} 19-\mathrm{C} 21$ & $1.512(5)$ & & \\
\hline $\mathrm{O} 1-\mathrm{Eu} 1-\mathrm{O} 5$ & $75.01(11)$ & $\mathrm{C} 22-\mathrm{C} 21-\mathrm{C} 19$ & $118.8(4)$ \\
\hline $\mathrm{O} 1-\mathrm{Eu} 1-\mathrm{N} 1$ & $145.99(10)$ & $\mathrm{C} 34-\mathrm{C} 21-\mathrm{C} 19$ & $121.1(4)$ \\
\hline $\mathrm{O} 1-\mathrm{Eu} 1-\mathrm{N} 3$ & $142.63(10)$ & $\mathrm{C} 34-\mathrm{C} 21-\mathrm{C} 22$ & $120.0(4)$ \\
\hline O1-Eu1-N5 & $118.84(11)$ & $\mathrm{C} 21-\mathrm{C} 22-\mathrm{C} 27$ & $119.9(5)$ \\
\hline $\mathrm{O} 2-\mathrm{Eu} 1-\mathrm{O} 1$ & $70.86(9)$ & $\mathrm{C} 23-\mathrm{C} 22-\mathrm{C} 21$ & $122.8(4)$ \\
\hline $\mathrm{O} 2-\mathrm{Eu} 1-\mathrm{O} 3$ & $145.67(9)$ & $\mathrm{C} 23-\mathrm{C} 22-\mathrm{C} 27$ & $117.3(5)$ \\
\hline $\mathrm{O} 2-\mathrm{Eu} 1-\mathrm{O} 4$ & $86.42(12)$ & $\mathrm{C} 22-\mathrm{C} 23-\mathrm{H} 23$ & 118.8 \\
\hline $\mathrm{O} 2-\mathrm{Eu} 1-\mathrm{O} 5$ & $109.01(12)$ & $\mathrm{C} 24-\mathrm{C} 23-\mathrm{C} 22$ & $122.3(5)$ \\
\hline $\mathrm{O} 2-\mathrm{Eu} 1-\mathrm{N} 1$ & $140.38(10)$ & $\mathrm{C} 24-\mathrm{C} 23-\mathrm{H} 23$ & 118.8 \\
\hline $\mathrm{O} 2-\mathrm{Eu} 1-\mathrm{N} 3$ & $79.35(11)$ & $\mathrm{C} 23-\mathrm{C} 24-\mathrm{H} 24$ & 120.0 \\
\hline $\mathrm{O} 2-\mathrm{Eu} 1-\mathrm{N} 5$ & $72.80(11)$ & $\mathrm{C} 23-\mathrm{C} 24-\mathrm{C} 25$ & $120.1(7)$ \\
\hline $\mathrm{O} 3-\mathrm{Eu} 1-\mathrm{O} 1$ & $79.81(9)$ & $\mathrm{C} 25-\mathrm{C} 24-\mathrm{H} 24$ & 120.0 \\
\hline $\mathrm{O} 3-\mathrm{Eu} 1-\mathrm{O} 5$ & $79.12(10)$ & $\mathrm{C} 24-\mathrm{C} 25-\mathrm{H} 25$ & 119.9 \\
\hline $\mathrm{O} 3-\mathrm{Eu} 1-\mathrm{N} 1$ & $73.13(9)$ & $\mathrm{C} 26-\mathrm{C} 25-\mathrm{C} 24$ & $120.3(6)$ \\
\hline $\mathrm{O} 3-\mathrm{Eu} 1-\mathrm{N} 3$ & $117.69(11)$ & $\mathrm{C} 26-\mathrm{C} 25-\mathrm{H} 25$ & 119.9 \\
\hline $\mathrm{O} 3-\mathrm{Eu} 1-\mathrm{N} 5$ & $139.36(10)$ & $\mathrm{C} 25-\mathrm{C} 26-\mathrm{H} 26$ & 119.1 \\
\hline $\mathrm{O} 4-\mathrm{Eu} 1-\mathrm{O} 1$ & $80.32(11)$ & $\mathrm{C} 25-\mathrm{C} 26-\mathrm{C} 27$ & $121.8(5)$ \\
\hline $\mathrm{O} 4-\mathrm{Eu} 1-\mathrm{O} 3$ & $71.10(9)$ & $\mathrm{C} 27-\mathrm{C} 26-\mathrm{H} 26$ & 119.1 \\
\hline $\mathrm{O} 4-\mathrm{Eu} 1-\mathrm{O} 5$ & $144.11(10)$ & $\mathrm{C} 26-\mathrm{C} 27-\mathrm{C} 22$ & $118.2(6)$ \\
\hline $\mathrm{O} 4-\mathrm{Eu} 1-\mathrm{N} 1$ & $109.05(11)$ & $\mathrm{C} 28-\mathrm{C} 27-\mathrm{C} 22$ & $119.1(5)$ \\
\hline $\mathrm{O} 4-\mathrm{Eu} 1-\mathrm{N} 3$ & $75.70(10)$ & $\mathrm{C} 28-\mathrm{C} 27-\mathrm{C} 26$ & $122.7(5)$ \\
\hline $\mathrm{O} 4-\mathrm{Eu} 1-\mathrm{N} 5$ & $143.07(11)$ & $\mathrm{C} 27-\mathrm{C} 28-\mathrm{H} 28$ & 118.6 \\
\hline $\mathrm{O} 5-\mathrm{Eu} 1-\mathrm{N} 1$ & $79.92(11)$ & $\mathrm{C} 27-\mathrm{C} 28-\mathrm{C} 29$ & $122.8(4)$ \\
\hline $\mathrm{O} 5-\mathrm{Eu} 1-\mathrm{N} 3$ & $137.76(10)$ & $\mathrm{C} 29-\mathrm{C} 28-\mathrm{H} 28$ & 118.6 \\
\hline $\mathrm{O} 5-\mathrm{Eu} 1-\mathrm{N} 5$ & $72.66(11)$ & $\mathrm{C} 28-\mathrm{C} 29-\mathrm{C} 30$ & $123.2(6)$ \\
\hline N3-Eu1-N1 & $70.18(10)$ & $\mathrm{C} 28-\mathrm{C} 29-\mathrm{C} 34$ & $119.0(5)$ \\
\hline N3-Eu1-N5 & $70.74(11)$ & $\mathrm{C} 30-\mathrm{C} 29-\mathrm{C} 34$ & $117.8(6)$ \\
\hline N5-Eu1-N1 & $73.60(11)$ & $\mathrm{C} 29-\mathrm{C} 30-\mathrm{H} 30$ & 119.4 \\
\hline $\mathrm{C} 1-\mathrm{O} 1-\mathrm{Eu} 1$ & $137.5(2)$ & $\mathrm{C} 31-\mathrm{C} 30-\mathrm{C} 29$ & $121.2(6)$ \\
\hline
\end{tabular}




\begin{tabular}{|c|c|}
\hline $\mathrm{C} 3-\mathrm{O} 2-\mathrm{Eu} 1$ & $139.3(3)$ \\
\hline $\mathrm{C} 18-\mathrm{O} 3-\mathrm{Eu} 1$ & $135.7(2)$ \\
\hline $\mathrm{C} 20-\mathrm{O} 4-\mathrm{Eu} 1$ & $135.2(3)$ \\
\hline $\mathrm{C} 48-\mathrm{O} 5-\mathrm{Eu} 1$ & $134.9(3)$ \\
\hline $\mathrm{N} 2-\mathrm{N} 1-\mathrm{Eu} 1$ & $125.2(2)$ \\
\hline $\mathrm{C} 40-\mathrm{N} 1-\mathrm{Eu} 1$ & $128.1(3)$ \\
\hline $\mathrm{C} 40-\mathrm{N} 1-\mathrm{N} 2$ & $105.9(3)$ \\
\hline $\mathrm{N} 1-\mathrm{N} 2-\mathrm{B} 1$ & $121.4(3)$ \\
\hline $\mathrm{C} 38-\mathrm{N} 2-\mathrm{N} 1$ & $110.1(3)$ \\
\hline $\mathrm{C} 38-\mathrm{N} 2-\mathrm{B} 1$ & $128.4(3)$ \\
\hline N4-N3-Eu1 & $125.2(2)$ \\
\hline $\mathrm{C} 41-\mathrm{N} 3-\mathrm{Eu} 1$ & $128.2(3)$ \\
\hline $\mathrm{C} 41-\mathrm{N} 3-\mathrm{N} 4$ & $106.1(3)$ \\
\hline $\mathrm{N} 3-\mathrm{N} 4-\mathrm{B} 1$ & $122.4(3)$ \\
\hline $\mathrm{C} 43-\mathrm{N} 4-\mathrm{N} 3$ & $109.8(3)$ \\
\hline $\mathrm{C} 43-\mathrm{N} 4-\mathrm{B} 1$ & $127.7(4)$ \\
\hline N6-N5-Eu1 & $125.4(2)$ \\
\hline $\mathrm{C} 35-\mathrm{N} 5-\mathrm{Eu} 1$ & $126.3(3)$ \\
\hline $\mathrm{C} 35-\mathrm{N} 5-\mathrm{N} 6$ & $106.6(4)$ \\
\hline $\mathrm{N} 5-\mathrm{N} 6-\mathrm{B} 1$ & $121.6(3)$ \\
\hline $\mathrm{C} 37-\mathrm{N} 6-\mathrm{N} 5$ & 109.9 (4) \\
\hline $\mathrm{C} 37-\mathrm{N} 6-\mathrm{B} 1$ & $128.4(4)$ \\
\hline $\mathrm{C} 48-\mathrm{N} 7-\mathrm{C} 49$ & $119.4(7)$ \\
\hline $\mathrm{C} 48-\mathrm{N} 7-\mathrm{C} 50$ & $122.5(6)$ \\
\hline $\mathrm{C} 50-\mathrm{N} 7-\mathrm{C} 49$ & $118.0(7)$ \\
\hline $\mathrm{O} 1-\mathrm{C} 1-\mathrm{C} 2$ & $125.6(4)$ \\
\hline $\mathrm{O} 1-\mathrm{C} 1-\mathrm{C} 45$ & $116.1(3)$ \\
\hline $\mathrm{C} 2-\mathrm{C} 1-\mathrm{C} 45$ & $118.2(3)$ \\
\hline $\mathrm{C} 1-\mathrm{C} 2-\mathrm{C} 4$ & $120.3(3)$ \\
\hline $\mathrm{C} 3-\mathrm{C} 2-\mathrm{C} 1$ & 120.5 \\
\hline $\mathrm{C} 3-\mathrm{C} 2-\mathrm{C} 4$ & $119.2(3)$ \\
\hline $\mathrm{O} 2-\mathrm{C} 3-\mathrm{C} 2$ & 124.9 (4) \\
\hline $\mathrm{O} 2-\mathrm{C} 3-\mathrm{C} 44$ & $116.1(4)$ \\
\hline $\mathrm{C} 2-\mathrm{C} 3-\mathrm{C} 44$ & $119.1(4)$ \\
\hline $\mathrm{C} 5-\mathrm{C} 4-\mathrm{C} 2$ & $120.4(4)$ \\
\hline $\mathrm{C} 17-\mathrm{C} 4-\mathrm{C} 2$ & $120.1(4)$ \\
\hline $\mathrm{C} 17-\mathrm{C} 4-\mathrm{C} 5$ & $119.5(4)$ \\
\hline $\mathrm{C} 4-\mathrm{C} 5-\mathrm{C} 10$ & $118.9(5)$ \\
\hline $\mathrm{C} 6-\mathrm{C} 5-\mathrm{C} 4$ & $123.2(4)$ \\
\hline $\mathrm{C} 6-\mathrm{C} 5-\mathrm{C} 10$ & $117.9(5)$ \\
\hline $\mathrm{C} 5-\mathrm{C} 6-\mathrm{H} 6$ & 118.8 \\
\hline $\mathrm{C} 7-\mathrm{C} 6-\mathrm{C} 5$ & $122.3(5)$ \\
\hline $\mathrm{C} 7-\mathrm{C} 6-\mathrm{H} 6$ & 118.8 \\
\hline $\mathrm{C} 6-\mathrm{C} 7-\mathrm{H} 7$ & 120.5 \\
\hline $\mathrm{C} 6-\mathrm{C} 7-\mathrm{C} 8$ & $119.0(7)$ \\
\hline $\mathrm{C} 8-\mathrm{C} 7-\mathrm{H} 7$ & 120.5 \\
\hline $\mathrm{C} 7-\mathrm{C} 8-\mathrm{H} 8$ & 119.7 \\
\hline $\mathrm{C} 9-\mathrm{C} 8-\mathrm{C} 7$ & $120.7(6)$ \\
\hline
\end{tabular}

\begin{tabular}{|c|c|}
\hline $\mathrm{C} 31-\mathrm{C} 30-\mathrm{H} 30$ & 119.4 \\
\hline $\mathrm{C} 30-\mathrm{C} 31-\mathrm{H} 31$ & 118.9 \\
\hline $\mathrm{C} 30-\mathrm{C} 31-\mathrm{C} 32$ & $122.3(6)$ \\
\hline $\mathrm{C} 32-\mathrm{C} 31-\mathrm{H} 31$ & 118.9 \\
\hline $\mathrm{C} 31-\mathrm{C} 32-\mathrm{H} 32$ & 121.1 \\
\hline $\mathrm{C} 33-\mathrm{C} 32-\mathrm{C} 31$ & $117.9(7)$ \\
\hline $\mathrm{C} 33-\mathrm{C} 32-\mathrm{H} 32$ & 121.1 \\
\hline $\mathrm{C} 32-\mathrm{C} 33-\mathrm{H} 33$ & 118.9 \\
\hline $\mathrm{C} 32-\mathrm{C} 33-\mathrm{C} 34$ & $122.3(6)$ \\
\hline $\mathrm{C} 34-\mathrm{C} 33-\mathrm{H} 33$ & 118.9 \\
\hline $\mathrm{C} 21-\mathrm{C} 34-\mathrm{C} 29$ & $119.1(5)$ \\
\hline $\mathrm{C} 21-\mathrm{C} 34-\mathrm{C} 33$ & $122.3(4)$ \\
\hline $\mathrm{C} 33-\mathrm{C} 34-\mathrm{C} 29$ & $118.6(5)$ \\
\hline N5-C35-H35 & 124.6 \\
\hline $\mathrm{N} 5-\mathrm{C} 35-\mathrm{C} 36$ & $110.9(6)$ \\
\hline C36-C35-H35 & 124.6 \\
\hline $\mathrm{C} 35-\mathrm{C} 36-\mathrm{H} 36$ & 127.6 \\
\hline $\mathrm{C} 35-\mathrm{C} 36-\mathrm{C} 37$ & $104.8(5)$ \\
\hline $\mathrm{C} 37-\mathrm{C} 36-\mathrm{H} 36$ & 127.6 \\
\hline $\mathrm{N} 6-\mathrm{C} 37-\mathrm{C} 36$ & $107.8(5)$ \\
\hline N6-C37-H37 & 126.1 \\
\hline $\mathrm{C} 36-\mathrm{C} 37-\mathrm{H} 37$ & 126.1 \\
\hline $\mathrm{N} 2-\mathrm{C} 38-\mathrm{H} 38$ & 125.8 \\
\hline $\mathrm{N} 2-\mathrm{C} 38-\mathrm{C} 39$ & $108.5(4)$ \\
\hline $\mathrm{C} 39-\mathrm{C} 38-\mathrm{H} 38$ & 125.8 \\
\hline $\mathrm{C} 38-\mathrm{C} 39-\mathrm{H} 39$ & 127.7 \\
\hline $\mathrm{C} 38-\mathrm{C} 39-\mathrm{C} 40$ & $104.5(4)$ \\
\hline $\mathrm{C} 40-\mathrm{C} 39-\mathrm{H} 39$ & 127.7 \\
\hline $\mathrm{N} 1-\mathrm{C} 40-\mathrm{C} 39$ & $110.9(4)$ \\
\hline $\mathrm{N} 1-\mathrm{C} 40-\mathrm{H} 40$ & 124.5 \\
\hline $\mathrm{C} 39-\mathrm{C} 40-\mathrm{H} 40$ & 124.5 \\
\hline $\mathrm{N} 3-\mathrm{C} 41-\mathrm{H} 41$ & 124.5 \\
\hline $\mathrm{N} 3-\mathrm{C} 41-\mathrm{C} 42$ & $111.0(4)$ \\
\hline $\mathrm{C} 42-\mathrm{C} 41-\mathrm{H} 41$ & 124.5 \\
\hline $\mathrm{C} 41-\mathrm{C} 42-\mathrm{H} 42$ & 127.7 \\
\hline $\mathrm{C} 43-\mathrm{C} 42-\mathrm{C} 41$ & $104.6(4)$ \\
\hline $\mathrm{C} 43-\mathrm{C} 42-\mathrm{H} 42$ & 127.7 \\
\hline $\mathrm{N} 4-\mathrm{C} 43-\mathrm{C} 42$ & $108.5(4)$ \\
\hline $\mathrm{N} 4-\mathrm{C} 43-\mathrm{H} 43$ & 125.7 \\
\hline $\mathrm{C} 42-\mathrm{C} 43-\mathrm{H} 43$ & 125.7 \\
\hline $\mathrm{C} 3-\mathrm{C} 44-\mathrm{H} 44 \mathrm{~A}$ & 109.5 \\
\hline $\mathrm{C} 3-\mathrm{C} 44-\mathrm{H} 44 \mathrm{~B}$ & 109.5 \\
\hline $\mathrm{C} 3-\mathrm{C} 44-\mathrm{H} 44 \mathrm{C}$ & 109.5 \\
\hline $\mathrm{H} 44 \mathrm{~A}-\mathrm{C} 44-\mathrm{H} 44 \mathrm{~B}$ & 109.5 \\
\hline $\mathrm{H} 44 \mathrm{~A}-\mathrm{C} 44-\mathrm{H} 44 \mathrm{C}$ & 109.5 \\
\hline $\mathrm{H} 44 \mathrm{~B}-\mathrm{C} 44-\mathrm{H} 44 \mathrm{C}$ & 109.5 \\
\hline $\mathrm{C} 1-\mathrm{C} 45-\mathrm{H} 45 \mathrm{~A}$ & 109.5 \\
\hline $\mathrm{C} 1-\mathrm{C} 45-\mathrm{H} 45 \mathrm{~B}$ & 109.5 \\
\hline
\end{tabular}




\begin{tabular}{|c|c|}
\hline $\mathrm{C} 9-\mathrm{C} 8-\mathrm{H} 8$ & 119.7 \\
\hline $\mathrm{C} 8-\mathrm{C} 9-\mathrm{H} 9$ & 118.9 \\
\hline $\mathrm{C} 8-\mathrm{C} 9-\mathrm{C} 10$ & $122.1(6)$ \\
\hline $\mathrm{C} 10-\mathrm{C} 9-\mathrm{H} 9$ & 118.9 \\
\hline $\mathrm{C} 9-\mathrm{C} 10-\mathrm{C} 5$ & $118.0(6)$ \\
\hline $\mathrm{C} 11-\mathrm{C} 10-\mathrm{C} 5$ & $120.0(5)$ \\
\hline $\mathrm{C} 11-\mathrm{C} 10-\mathrm{C} 9$ & $122.0(5)$ \\
\hline $\mathrm{C} 10-\mathrm{C} 11-\mathrm{H} 11$ & 119.0 \\
\hline $\mathrm{C} 12-\mathrm{C} 11-\mathrm{C} 10$ & $122.0(4)$ \\
\hline $\mathrm{C} 12-\mathrm{C} 11-\mathrm{H} 11$ & 119.0 \\
\hline $\mathrm{C} 11-\mathrm{C} 12-\mathrm{C} 13$ & $122.9(5)$ \\
\hline $\mathrm{C} 11-\mathrm{C} 12-\mathrm{C} 17$ & $119.0(5)$ \\
\hline $\mathrm{C} 13-\mathrm{C} 12-\mathrm{C} 17$ & $118.1(6)$ \\
\hline $\mathrm{C} 12-\mathrm{C} 13-\mathrm{H} 13$ & 118.9 \\
\hline $\mathrm{C} 14-\mathrm{C} 13-\mathrm{C} 12$ & $122.2(6)$ \\
\hline $\mathrm{C} 14-\mathrm{C} 13-\mathrm{H} 13$ & 118.9 \\
\hline $\mathrm{C} 13-\mathrm{C} 14-\mathrm{H} 14$ & 120.1 \\
\hline $\mathrm{C} 13-\mathrm{C} 14-\mathrm{C} 15$ & $119.7(6)$ \\
\hline $\mathrm{C} 15-\mathrm{C} 14-\mathrm{H} 14$ & 120.1 \\
\hline $\mathrm{C} 14-\mathrm{C} 15-\mathrm{H} 15$ & 119.5 \\
\hline $\mathrm{C} 16-\mathrm{C} 15-\mathrm{C} 14$ & $120.9(7)$ \\
\hline $\mathrm{C} 16-\mathrm{C} 15-\mathrm{H} 15$ & 119.5 \\
\hline $\mathrm{C} 15-\mathrm{C} 16-\mathrm{H} 16$ & 119.5 \\
\hline $\mathrm{C} 15-\mathrm{C} 16-\mathrm{C} 17$ & $121.1(6)$ \\
\hline $\mathrm{C} 17-\mathrm{C} 16-\mathrm{H} 16$ & 119.5 \\
\hline $\mathrm{C} 4-\mathrm{C} 17-\mathrm{C} 12$ & $120.6(5)$ \\
\hline $\mathrm{C} 4-\mathrm{C} 17-\mathrm{C} 16$ & $121.5(4)$ \\
\hline $\mathrm{C} 16-\mathrm{C} 17-\mathrm{C} 12$ & $117.9(5)$ \\
\hline $\mathrm{O} 3-\mathrm{C} 18-\mathrm{C} 19$ & 124.9 (3) \\
\hline $\mathrm{O} 3-\mathrm{C} 18-\mathrm{C} 47$ & $115.2(3)$ \\
\hline $\mathrm{C} 19-\mathrm{C} 18-\mathrm{C} 47$ & $120.0(3)$ \\
\hline $\mathrm{C} 18-\mathrm{C} 19-\mathrm{C} 21$ & $118.2(3)$ \\
\hline $\mathrm{C} 20-\mathrm{C} 19-\mathrm{C} 18$ & $122.0(3)$ \\
\hline $\mathrm{C} 20-\mathrm{C} 19-\mathrm{C} 21$ & $119.8(3)$ \\
\hline $\mathrm{O} 4-\mathrm{C} 20-\mathrm{C} 19$ & $125.1(3)$ \\
\hline $\mathrm{O} 4-\mathrm{C} 20-\mathrm{C} 46$ & $115.7(4)$ \\
\hline $\mathrm{C} 19-\mathrm{C} 20-\mathrm{C} 46$ & $119.3(3)$ \\
\hline
\end{tabular}

$\begin{array}{ll}\text { C1-C45-H45C } & 109.5 \\ \text { H45A-C45-H45B } & 109.5 \\ \text { H45A-C45-H45C } & 109.5 \\ \text { H45B-C45-H45C } & 109.5 \\ \text { C20-C46-H46A } & 109.5 \\ \text { C20-C46-H46B } & 109.5 \\ \text { C20-C46-H46C } & 109.5 \\ \text { H46A-C46-H46B } & 109.5 \\ \text { H46A-C46-H46C } & 109.5 \\ \text { H46B-C46-H46C } & 109.5 \\ \text { C18-C47-H47A } & 109.5 \\ \text { C18-C47-H47B } & 109.5 \\ \text { C18-C47-H47C } & 109.5 \\ \text { H47A-C47-H47B } & 109.5 \\ \text { H47A-C47-H47C } & 109.5 \\ \text { H47B-C47-H47C } & 109.5 \\ \text { O5-C48-N7 } & 124.7(6) \\ \text { O5-C48-H48 } & 117.6 \\ \text { N7-C48-H48 } & 117.6 \\ \text { N7-C49-H49A } & 109.5 \\ \text { N7-C49-H49B } & 109.5 \\ \text { N7-C49-H49C } & 109.5 \\ \text { H49A-C49-H49B } & 109.5 \\ \text { H49A-C49-H49C } & 109.5 \\ \text { H49B-C49-H49C } & 109.5 \\ \text { N7-C50-H50A } & 109.5 \\ \text { N7-C50-H50B } & 109.5 \\ \text { N7-C50-H50C } & 109.5 \\ \text { H50A-C50-H50B } & 109.5 \\ \text { H50A-C50-H50C } & 109.5 \\ \text { H50B-C50-H50C } & 109.5 \\ \text { N2-B1-H1 } & 109.9 \\ \text { N4-B1-N2 } & 109.6(3) \\ \text { N4-B1-N6 } & 109.7(3) \\ \text { N4-B1-H1 } & 109.9 \\ \text { N6-B1-N2 } & 107.9(4) \\ \text { N6-B1-H1 } & 109.9 \\ & \end{array}$

